\title{
Reevaluación de intensidades macrosísmicas del terremoto del 07 de junio de 1925 en Colombia
}

\author{
Elkin de Jesús Salcedo-Hurtado ${ }^{1,2^{*}} \mathbb{D}$; Juan Camilo Blandón-Silva ${ }^{2} \mathbb{D}$; Luisa Ríos-García ${ }^{2}$
}

Forma de citar: Salcedo-Hurtado, E. de J.; Blandón-Silva, J.C.; Ríos-García, L. (2021). Reevaluación de intensidades macrosísmicas del terremoto del 07 de junio de 1925 en Colombia. Boletín de Geología, 43(3), 35-61. https://doi.org/10.18273/revbol.v43n3-2021002

\begin{abstract}
Resumen
El sismo ocurrido el 07 de junio de 1925 es un evento sentido en varias regiones de Colombia, cuya severidad de sus efectos es bastante considerable. Varios autores han realizado investigaciones que contribuyen a mejorar el conocimiento de este evento sísmico. En el presente trabajo se contrastan las hipótesis de intensidades dadas en trabajos anteriores, mostrándose la disimilitud en el uso de las escalas macrosísmicas y la falta de correlación entre los valores de intensidad macrosísmica asignados. Se manifiesta el hallazgo de nuevas noticias sísmicas que, por un lado, amplían el número de poblaciones afectadas por este terremoto, y, por el otro, mejoran la información sobre los daños y efectos mostrados en otras poblaciones, de tal manera que se mejora la calidad de la información para proponer nuevos valores de intensidad más precisos y llenar vacíos espaciales geográficos. La intensidad macrosísmica ha sido evaluada en la escala EMS-98. La distribución geográfica de los puntos de intensidad marca una ligera tendencia SSO-NNE, donde las mayores intensidades se asignan a poblaciones del departamento del Valle del Cauca, con valores que van desde V hasta VIII; la máxima intensidad se reporta en la ciudad de Cali. A partir de aquí, se aprecia el decaimiento de la intensidad tanto hacia el NNE como hacia el SSO, con mayor elongación hacia el NNE. El epicentro macrosísmico se ubica en las coordenadas $3,45^{\circ}$ de latitud norte y $76,49^{\circ}$ de longitud oeste.
\end{abstract}

Palabras clave: Datos macrosísmicos; Intensidad macrosísmica; Escala EMS-98; Mapa de isosistas.

\section{Re-assessment of macroseismic intensities of the earthquake of June 7, 1925 in Colombia}

\begin{abstract}
The earthquake occurred on June 07 of 1925 is an event felt in several regions of Colombia, the harshness of its effects was quite considerable. Many authors have carried out research that contributes to improving the knowledge of this seismic event. The hypotheses of intensities that were given in previous works are contrasted in this one, showing the dissimilarity in the use of macroseismic scales and the lack of correlation in the assigned macroseismic intensity values. The finding of new historical accounts on the earthquake reflects on the increasing number of inhabitants affected by this earthquake, as well as on improving the information about the damages and effects shown in other populations; therefore, the information quality is improved in order to propose new more precise intensity values, which also fill empty geographical gaps. The macroseismic data has been evaluated in the EMS-98 scale. The geographic distribution of the intensity points highlights a slightly SSW-NNE trend, where the highest intensities are assigned to settlements in department of Valle del Cauca, with values ranging from V to VIII, being the highest intensity reported in Cali city. From this, the decay of the intensity is appreciated on both directions, towards the NNE and the SSW, with greater elongation towards the NNE. The macroseismic epicenter is located at the coordinates latitude $3.45^{\circ}$ North and longitude $76.49^{\circ}$ West.
\end{abstract}

Keywords: Macroseismic dates; Macroseismic intensity; EM-98 scale; Isoseismals map.

\footnotetext{
${ }^{1}$ Departamento de Geografía, Universidad del Valle, Santiago de Cali, Colombia. (*) elkin.salcedo@correounivalle.edu.co ${ }^{2}$ Grupo Georiesgos, Observatorio Sismológico y Geofísico del Suroccidente Colombiano, Universidad del Valle, Santiago de Cali, Colombia. juancho9412@hotmail.com; luisa.rios.garcia@correounivalle.edu.co
} 


\section{Introducción}

La región del suroccidente de Colombia es una de las zonas de mayor actividad sísmica en el país, por lo cual ha sido escenario de múltiples eventos sísmicos históricos y recientes; sin embargo, la valoración de las intensidades que allí tienen lugar no se realiza con la misma proporción de la actividad sísmica, lo cual deja vacíos considerables en los catálogos sismológicos, y al mismo tiempo reduce la efectividad de los estudios de amenaza, vulnerabilidad y riesgo sísmico en la región. Uno de los eventos sísmicos importantes ocurridos en esta región, es el terremoto del 07 de junio de 1925, que causó daños considerables a poblaciones de diferentes departamentos como Valle del Cauca, Cauca, Tolima, Cundinamarca, entre otros.

El primer reporte sobre este evento lo marca instrumentalmente Gutenberg y Richter (1954), quienes registran su ocurrencia a las 23:41 UTC, en el océano Pacífico en las coordenadas $3,0^{\circ} \mathrm{N}$ de latitud y $78,0^{\circ} \mathrm{O}$ de longitud, a $170 \mathrm{~km}$ de profundidad. Engdahl y Villaseñor (2002) establecen que el sismo tuvo su epicentro cerca del municipio de Tuluá en el departamento del Valle del Cauca, con coordenadas $4,02^{\circ} \mathrm{N}$ de latitud y $76,07^{\circ} \mathrm{O}$ de longitud, profundidad superficial, establecida convencionalmente en $33 \mathrm{~km}$. El proyecto Global Earthquake Model (ISG-GEM, 2015) asigna las coordenadas del epicentro en $4,279^{\circ} \mathrm{N}$ y $75,331^{\circ} \mathrm{O}$, y magnitud $\mathrm{Mw} 6,3$. La incertidumbre en la determinación de las coordenadas epicentrales y la profundidad no ha permitido su asignación a una fuente sismogénica concreta. En la Figura 1 se presenta, sobre el mapa geológico del departamento del Valle del Cauca el epicentro macrosísmico del terremoto del 07 de junio de 1925; también se muestran las principales fallas activas de la región, como Río Bravo, Dagua-Calima, Roldanillo, Palmira-Buga, Silvia-Pijao y los sistemas de falla Cali-Patía y CaucaAlmaguer, que en general mantienen una dirección SSO-NNE (París y Romero, 1994; París et al., 2000; Nivia, 2001; INGEOMINAS, 2005b). Algunas de estas fallas han sido responsables de importantes eventos sísmicos históricos que han causado daños o efectos considerables en varias poblaciones del suroccidente colombiano. En el presente estudio el epicentro macrosísmico, preliminarmente, se ubica cerca de la ciudad de Cali, y podría estar asociado al sistema de fallas Cali-Patía, en una zona de planicie aluvial, lo cual deberá corroborarse al establecer su profundidad.

Para este evento se han realizado varios estudios macrosísmicos utilizando diversas escalas para la asignación de intensidades (Espinosa-Baquero, 2003; INGEOMINAS, 2005a, 2010; Salcedo-Hurtado et al., 2007; Martínez, 2014). Debido a la diversidad de escalas usadas, las hipótesis de intensidad muestran que los resultados obtenidos hasta ahora presentan discrepancias. Por otro lado, estos estudios no hacen una valoración homogénea en todas las poblaciones donde las fuentes documentales reportan algún tipo de daño, por tanto, dejan vacíos geográficos en la información.

Sin embargo, los estudios macrosísmicos coinciden en que los mayores efectos se presentaron en la ciudad de Cali, donde ocurrió la destrucción de la iglesia de las monjas de la Encarnación, además de daños mayores en las iglesias Matriz, Santo Domingo, La Merced, San Agustín, San Francisco y daños considerables en la Ermita (INGEOMINAS, 2005a).

Por su importancia en la amenaza sísmica regional, se hace necesario la revisión de la información y la reevaluación de las intensidades macrosísmicas para este evento, pues, como se ha mencionado, diversos autores han propuesto valores de intensidad cuya calidad es bastante heterogénea debido al análisis que han realizado a la luz de diversas escalas macrosísmicas.

Ante esta situación, dado que para los estudios de amenaza y riesgo sísmico se requieren catálogos confiables, Guidoboni y Stucchi (1993) señalan que se puede elegir entre dos posiciones: mantener lo disponible como lo mejor posible o realizar una nueva investigación que mejore el conocimiento del sismo escogido.

El presente estudio opta por la segunda posición, es decir, continuar la búsqueda de más fuentes que aporten nuevos datos o precisen los ya existentes, con el propósito de mejorar el conocimiento de las particularidades del terremoto del 07 de junio de 1925, empezando, como lo proponen Guidoboni y Stucchi (1993), por volver a analizar y explotar todos los datos que ya están disponibles en las compilaciones sismológicas anteriores.

Así, se realiza la reevaluación de la intensidad macrosísmica del terremoto del 07 de junio de 1925, aplicando el método intensivista (Rodríguez de la Torre, 1993), que permite conseguir nuevas noticias y datos macrosísmicos en algunas poblaciones afectadas que no son reportados por los estudios previos. De igual manera, se hace la clasificación de las fuentes bibliográficas determinando el respectivo "árbol genealógico" de los estudios realizados, y se discriminan los efectos causados aplicando los criterios de la Escala Macrosísmica 
Europea 1998 (EMS-98 por sus siglas en inglés), de tal manera que se unifica la asignación de intensidades. La intensidad asignada en cada población introduce el factor Q que valora la calidad de la información de las fuentes bibliográficas usadas.
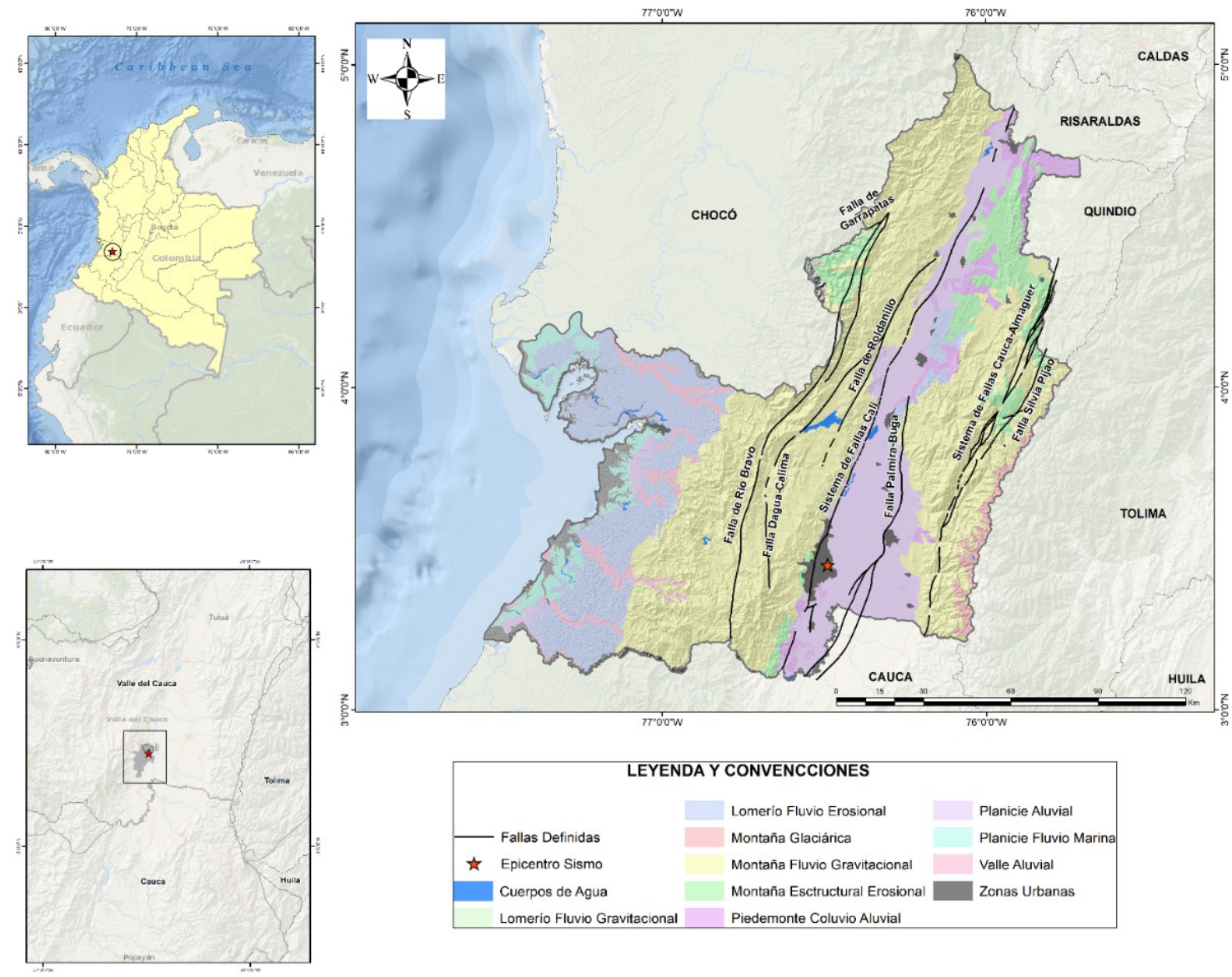

Figura 1. Mapa geológico del departamento del Valle del Cauca (tomado de CVC, 2019), donde se muestra el epicentro del sismo del 07 de junio de 1925, el cual se demarca con una estrella roja.

\section{Marco teórico}

\section{Sismicidad histórica}

Uno de los problemas que se presenta en la evaluación de la amenaza sísmica en una región determinada es la falta de información que permita en un largo periodo, por un lado, determinar el sismo característico o evento máximo posible $\mathrm{y}$, por otro lado, calcular el periodo de retorno o tiempo promedio en el cual podría presentarse dicho evento. Para resolver este problema, se requiere realizar estudios confiables de sismicidad histórica que conduzcan al mejor conocimiento de los terremotos que han ocurrido, al ampliar la ventana de observación sismológica al reciente histórico del país; entiéndase para Colombia, a partir de la conquista española a comienzos del siglo XVI.
Los estudios históricos tienen la finalidad de realizar la búsqueda de diversos tipos de información (observaciones de campo, relatos detectados en fuentes escritas como manuscritos, libros y prensa o, incluso, instrumental) sobre los daños y efectos causados por un terremoto en un área específica. Dependiendo de la historia sísmica que tenga la región donde se pretenda llevar a cabo un estudio sobre los sismos ocurridos en tiempos lejanos y del tipo de información a consultar, según Gouin (1994), Audemard (1998, 2003, 2005) y Salcedo-Hurtado (1999), los periodos de análisis para los estudios históricos sobre terremotos pueden ser divididos en tres tipos:

- Periodo paleo o fósil sismicidad: en este se tiene en cuenta que los registros de los terremotos 
siempre dejan huellas en el terreno, especialmente, en las zonas de las fallas geológicas, por lo cual se requiere realizar estudios paleosismológicos para determinar la falla geológica generadora y, además, las características de los terremotos que pudieron haber ocurrido antes de contar con las crónicas o relatos escritos; en muchos casos, este periodo puede ser de más de 2.000 años. Sin embargo, los estudios paleosismológicos también han sido efectivos para determinar parámetros como la magnitud de eventos grandes ocurridos en periodos no instrumentales, como es el caso del sismo del 28 de abril de 1894, que tuvo lugar en un segmento de la falla Boconó en la zona de los Andes en territorio venezolano (Audemard, 1998; Audemard et al., 2008).

- Periodo histórico: en este caso, los efectos de los terremotos se registran en diversos tipos de documentos que pueden ser localizados en diferentes fondos o libros de los archivos históricos, bibliotecas, hemerotecas, entre otros. Generalmente, este tipo de información corresponde a narraciones hechas por autores no científicos, pero que pueden ser interpretadas de manera apropiada para precisar tiempo y lugar de los terremotos, así como para caracterizar los daños, lo que conduce a la valoración de la intensidad macrosísmica. En muchos países se encuentran numerosas publicaciones sobre historia de los terremotos (v.g. Ramírez, 1975), alimentadas con narrativas históricas sobre eventos sísmicos.

- Periodo instrumental: corresponde a la época en que ya se ha instalado instrumentación apropiada y suficiente para la determinación de los diversos parámetros que caracterizan los terremotos. En algunos países, desde comienzos del siglo veinte, se instalaron equipos sismológicos no calibrados, pero que con el correr del tiempo lograron obtener suficiente precisión sobre su sismicidad.

De esta manera, los datos históricos sobre terremotos aportan información sobre los daños ocasionados en diversas poblaciones, permitiendo obtener los datos cualitativos que son interpretados a la luz de las escalas macrosísmicas para evaluar el grado de intensidad en cada pueblo o localidad, conocido como centro o punto de intensidad; esto posibilita la elaboración de mapas de intensidades a partir de los cuales se trazarían, cuando se tienen puntos suficientes, las curvas de isosistas, que corresponden a curvas que encierran zonas de igual intensidad.

La investigación histórica, en especial la que corresponde al periodo histórico propiamente dicho, se desarrolla mediante la lectura sistemática de distintas fuentes, permitiendo ampliar el conocimiento sobre eventos símicos conocidos (investigación intensivista) o encontrar nuevos sismos no incluidos en los catálogos sismológicos (investigación extensivista) (Rodríguez de la Torre, 1993). En estos casos, es muy importante realizar búsqueda en diversos tipos de espacios como archivos históricos, archivos locales (municipales o de ciudades y notariales), archivos de iglesias y monasterios, archivos de dominios aristocráticos.

La información que se recoja en estos archivos tiene la ventaja de ser un material que corresponde a relatos de testigos oculares, y puede dar cuenta sobre daños en edificaciones y diferentes tipos de efectos en diversas áreas. Sin embargo, también tiene la desventaja de que estos archivos frecuentemente están en desorden: los investigadores pueden encontrar información solamente estudiando el archivo completo o por casualidad; igualmente, la información puede ser muy limitada y a veces dudosa (Salcedo-Hurtado, 1999), por lo cual se requiere de cierta experticia para la interpretación y para la adecuada asignación de la intensidad sísmica. En este sentido, Rodríguez y Audemard (2003) dan cuenta de un rosario de limitaciones que tiene esta fuente documental particular, con base en casos venezolanos estudiados por esta aproximación metodológica.

De acuerdo con Salcedo-Hurtado (1999), un estudio histórico sobre los terremotos requiere de la búsqueda de fuentes primarias, que permitan tener registros detallados tomados de fuentes confiables para que sean interpretadas con fines sismológicos. Stucchi (1994) señala que, para poder ser utilizados con fines de interpretaciones sismológicas, los registros históricos necesitan ser ubicados en el espacio y el tiempo, además, interpretados en términos de la intensidad macrosísmica; tal operación produce las llamadas intensidades puntuales (o centros de intensidad), las cuales pueden ser asumidas como observaciones macrosísmicas. Dichos datos de intensidad alimentan los catálogos sísmicos de una región. 


\section{Clasificación y valoración de las fuentes bibliográficas}

Un aspecto fundamental para la evaluación de intensidades macrosísmicas de terremotos históricos concierne a la valoración de las fuentes bibliográficas. Izquierdo-Álvarez (1999) considera que al valorar las fuentes de información se debe tener claro su tipo y finalidad (si se trata de un informe para pedir ayudas económicas o de un diario personal), fiabilidad, grado de originalidad (si la información es de primera mano o proviene de otra fuente); se debe ser cuidadoso para que la información que se extraiga de cada fuente sea lo más exacta posible, tratando de perder la menor cantidad de datos. Es importante tener mucho cuidado con la localización espacio-temporal, puesto que errores de esta clase pueden dar lugar a duplicaciones de eventos, a atribuir efectos a terremotos o lugares equivocados, entre otros aspectos.

\section{Intensidad y mapa de intensidad sísmica}

Existen diversos tipos de parámetros mediante los cuales se puede valorar la fuerza de un terremoto, entre ellos parámetros instrumentales y no instrumentales. Entre los instrumentales se encuentra la magnitud, que corresponde a una escala cuantitativa que inicialmente Charles Richter relacionó con la energía liberada por un sismo, que es independiente del lugar de observación (Lay y Wallace, 1995; Bolt, 2003). Por su parte, entre los parámetros no instrumentales más representativos está la intensidad sísmica, representada como I.

La intensidad sísmica se entiende como una unidad de medida adimensional que clasifica la severidad o las manifestaciones de las vibraciones sísmicas en la superficie terrestre; se mide en grados y se especifica con un número romano previamente determinado por una escala específica. La medición de la intensidad sísmica no requiere de instrumentos para ser evaluada; sin embargo, se hace a través de ciertos sensores usados históricamente, que pueden ser divididos en cuatro grupos (Grünthal, 2009):

- Seres vivos-gente y animales. Según aumenta la intensidad, una mayor proporción de gente o animales (a) nota las vibraciones y (b) se asusta debido a ellas.

- Objetos ordinarios. Según aumenta la intensidad, un mayor número de objetos domésticos ordinarios (utensilios de cocina, libros, etc.) empieza a vibrar, desordenarse y caer al suelo.
- Edificios. Según aumenta la intensidad, los edificios experimentan progresivamente mayores daños.

- El ambiente natural. Según aumenta la intensidad, existe mayor probabilidad de que se manifiesten efectos como grietas en diques, deslizamientos, caídas de rocas, etc.

El hecho de que la intensidad no sea medida instrumentalmente le da un carácter subjetivo a su valoración, la cual depende de cómo el observador interprete la información sobre los daños y efectos causados por terremotos, descritos acorde con los sensores indicados.

Existen diversas escalas para valorar el nivel de intensidad de un terremoto. En su mayoría, estas escalas estiman doce grados del I al XII, en los cuales I corresponde a vibraciones débiles que solo son notadas por los instrumentos, sin que sean sentidas por las personas; XII hace referencia a fuertes catástrofes, donde ninguna construcción se sostiene, hay grandes cambios en el relieve y muchas víctimas; desde el grado II hasta el XI se consideran como efectos intermedios. Entonces, se acostumbra designar los grados de intensidad con numeración que va desde I a XII, como es el caso de la Escala Macrosísmica Europea, que corresponde a una actualización de la escala MSK-64, realizada por la Comisión Europea en el año 1998 (Grünthal, 2009).

La evaluación de la intensidad sísmica de un terremoto muestra que es mayor en la zona epicentral (área de la superficie terrestre donde se observa la mayor cantidad y más severos daños causados por el terremoto) y disminuye con la distancia a media que el observador se aleja de la fuente sísmica. La intensidad evaluada en las vecindades del epicentro se conoce como la intensidad epicentral y se presenta como $\mathrm{I}_{0}$. A partir de este criterio, se pueden establecer modelos de atenuación de la intensidad, los cuales son fundamentales para la valoración de la amenaza y el riesgo sísmico.

La asignación del nivel de intensidad de un terremoto en diferentes sitios sobre la superficie terrestre conduce a la realización de los denominados mapas de intensidad. La elaboración de un mapa de intensidades conlleva a elegir sitios georreferenciados sobre la superficie de la Tierra, que han sido afectados por las ondas sísmicas generadas por un terremoto fuerte, $\mathrm{y}$ asociarles un valor de intensidad conforme a una escala macrosísmica elegida previamente. 


\section{Mapas de isosistas}

Un mapa de isosistas consiste en el trazado de curvas que encierran áreas de igual nivel de intensidad sísmica en una región que ha sido afectada durante la propagación de las ondas sísmicas generadas por un terremoto. A medida que las ondas sísmicas se alejan del epicentro, se espera un decrecimiento de su intensidad $\mathrm{y}$, dependiendo del tipo de terreno que atraviesen, su propagación se ve favorecida o retardada: hay mejor propagación en la dirección que transcurre la estructura de la fuente generadora del sismo, generalmente una falla geológica, y mayor atenuación en sentido perpendicular, razón por la cual las líneas de isosistas suelen tener forma de elipse relativamente deformada (Musson y Cecić, 2012). En algunos casos estas curvas se simplifican y tienden a dibujarse de manera circular; el caso es que la realización de las isosistas implica el trazo de isolíneas que encierran iguales valores de intensidad.

Generalmente, el epicentro macrosísmico de un terremoto está rodeado por la isosista de mayor valor, la cual bordea la zona pleistosísmica respondiendo, con alguna aproximación, a la forma del foco, que aparece como la última proyección en la superficie de la tierra. Dependiendo de la rapidez con que se atenúen las ondas sísmicas, en la medida que aumenta la distancia epicentral, se configura la distribución y forma de las isosistas subsiguientes, las cuales reflejan claramente los campos de emisión, transmisión y recepción de un terremoto.

Las curvas de isosistas han sido utilizadas con diversos propósitos en el campo de la sismología y la ingeniería y para establecer las áreas de mayor intervención en la mitigación y atención de los efectos causados por fuertes terremotos. Por ejemplo, Grases y Rodríguez (2001) establecen correlaciones que permiten calcular la magnitud de un sismo histórico (no-instrumental) a partir del tamaño o superficie de las áreas de una intensidad dada. De igual manera, como lo muestran Salcedo-Hurtado et al. (2021a), los mapas de isosistas también son usados para calcular diversos parámetros focales de los terremotos.

\section{Estudios anteriores}

El terremoto del 07 de junio de 1925 tuvo lugar a las 6:47 de la tarde; provocó efectos y daños serios en varias regiones de Colombia. Al respecto, se han realizado diferentes estudios que han hecho aportes significativos sobre un mejor conocimiento de las particularidades del suceso y la distribución geográfica de sus efectos. Dichos aportes pueden ser clasificados como información instrumental y estudios macrosísmicos.

\section{Información instrumental}

La primera fuente instrumental que da cuenta de este evento sísmico es el catálogo elaborado por Gutenberg y Richter (1954), que lo registra a las 23:41 UTC, localizado en el océano Pacífico en las coordenadas $3,0^{\circ} \mathrm{N}$ de latitud y $78,0^{\circ} \mathrm{O}$ de longitud, a $170 \mathrm{~km}$ de profundidad. Por su parte, Engdahl y Villaseñor (2002) establecen que el sismo tuvo su epicentro cerca del municipio de Tuluá, en el departamento del Valle del Cauca, con coordenadas de $4,02^{\circ} \mathrm{N}$ de latitud y $76,07^{\circ} \mathrm{O}$ de longitud, con profundidad superficial, convencionalmente establecida en $33 \mathrm{~km}$. En el catálogo de CERESIS (1985) se transcriben los parámetros instrumentales inicialmente reportados por Gutenberg y Richter. El proyecto Global Earthquake Model (ISG-GEM, 2015) se definen las coordenadas del epicentro en $4,279^{\circ} \mathrm{N}$ y $75,331^{\circ} \mathrm{O}$, y se calcula una magnitud Mw 6,3.

\section{Información y estudios macrosísmicos}

El primer reporte macrosísmico de este terremoto aparece en el informe presentado por el Grupo de Estudio de Riesgo Sísmico del Suroccidente Colombiano (GERSCO, 1987); estos datos son retomados y ampliados por Espinosa-Baquero (2003). El trabajo de Meyer (1990) también retoma los datos macrosísmicos de GERSCO y propone que el epicentro se localiza al norte de la ciudad de Cali, con origen en la zona de Wadatti-Benioff (entiéndase sismo de subducción), cambiando la localización previamente dada por Engdahl y Villaseñor (2002). Por su parte, Mendoza et al. (2004) reportan nuevos datos macrosísmicos que indican que los daños causados por este evento se concentran principalmente en poblaciones del departamento del Valle del Cauca. Las primeras hipótesis de intensidad macrosísmica son aportadas por Espinosa-Baquero (2003). Otros autores como INGEOMINAS (2005a, 2010), Salcedo-Hurtado et al. (2007) y Martínez (2014) han aportado nuevos datos para mejorar el conocimiento de los parámetros sismológicos de este evento sísmico. Cabe señalar que ninguna de las versiones del libro Historia de los terremotos en Colombia del padre Jesús Emilio Ramírez (Ramírez, 1975) incluye información sobre los efectos causados por este evento. 
En la Figura 2 se muestra la secuencia temporal y la naturaleza de los estudios anteriormente realizados sobre el terremoto del 07 de junio de 1925, ocurrido en el suroccidente colombiano. Por su parte, la Tabla 1 presenta las intensidades asignadas por cada uno de los estudios anteriores, acorde con la información recopilada. Como se aprecia, por causa de este terremoto resultaron afectados diversos municipios de varios departamentos de las regiones del Pacífico, Eje Cafetero, centro y norte de Colombia; sin embargo, los mayores efectos se presentaron en la ciudad de Cali y en otras poblaciones del departamento del Valle del Cauca.
Se puede apreciar que en el caso del estudio de Espinosa-Baquero (2003), se asigna intensidad de VIII a las poblaciones de Cali, La Cumbre, Restrepo y Yotoco. Los demás autores asignan un valor máximo de intensidad entre VII-VIII para la ciudad de Cali. Puede verse que no todas las poblaciones mencionadas en la Tabla 1 tienen asignaciones de intensidad, lo cual muestra un vacío de información macrosísmica que es complementado con la búsqueda de nueva información realizada en el presente estudio, como se muestra más adelante.

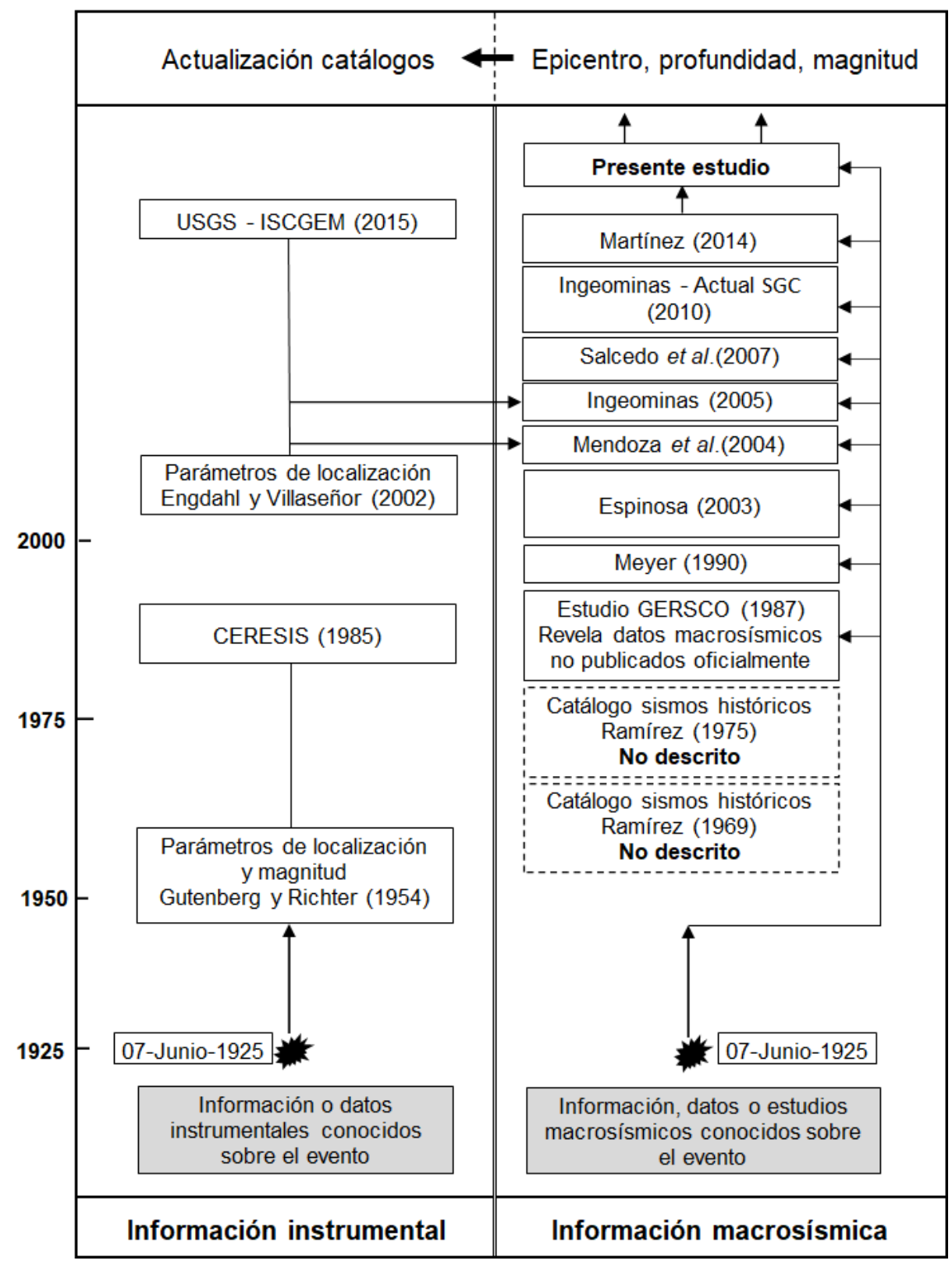

Figura 2. Estado del arte del terremoto del 07 de junio de 1925 (modificado de Salcedo-Hurtado et al., 2007). 
Reevaluación de intensidades macrosísmicas del terremoto del 07 de junio de 1925 en Colombia

Tabla 1. Intensidades del terremoto de 1925 asignadas en estudios anteriores.

\begin{tabular}{|c|c|c|c|c|c|c|}
\hline No. & $\begin{array}{l}\text { Población } \\
\text { afectada }\end{array}$ & Departamento & $\begin{array}{c}\text { Espinosa- } \\
\text { Baquero } \\
(2003) \\
(\mathrm{MM}) \\
\end{array}$ & $\begin{array}{c}\text { Salcedo- } \\
\text { Hurtado } \text { et al. } \\
(2007) \\
\text { EMS-92 }\end{array}$ & $\begin{array}{c}\text { INGEOMINAS } \\
\text { (2010) } \\
\text { EMS-98 }\end{array}$ & $\begin{array}{c}\text { Martínez } \\
\text { (2014) } \\
\text { EMS-98 }\end{array}$ \\
\hline 1 & Armenia & Quindío & $\mathrm{V}$ & --- & $\mathrm{V}$ & V \\
\hline 2 & Barranquilla & Atlántico & IV & --- & --- & --- \\
\hline 3 & Bogotá & Cundinamarca & IV & --- & IV & $\mathrm{V}$ \\
\hline 4 & Briceño & Boyacá & V & --- & --- & --- \\
\hline 5 & Buenaventura & Valle del Cauca & VI & $\mathrm{V}$ & $\mathrm{V}$ & IV \\
\hline 6 & Buga & Valle del Cauca & VII & VII & VI & VII \\
\hline 7 & Calarcá & Quindío & VI & $\mathrm{V}$ & V & V \\
\hline 8 & Cali & Valle del Cauca & VIII & VII-VIII & VII-VIII & VII-VIII \\
\hline 9 & Cértegui & Chocó & ---- & --- & --- & --- \\
\hline 10 & Chaparral & Tolima & $\mathrm{V}$ & --- & --- & --- \\
\hline 11 & Chicoral & Tolima & V & --- & --- & --- \\
\hline 12 & Condoto & Chocó & --- & --- & --- & --- \\
\hline 13 & Cúcuta & Norte de Santander & --- & --- & --- & --- \\
\hline 14 & El Cerrito & Valle del Cauca & --- & VII & VII & VII \\
\hline 15 & Facatativá & Cundinamarca & IV & --- & --- & --- \\
\hline 16 & Girardot & Cundinamarca & V & V & $\mathrm{V}$ & V \\
\hline 17 & Guamo & Tolima & IV & --- & --- & --- \\
\hline 18 & Ibagué & Tolima & VI & VI & $\mathrm{V}$ & VI \\
\hline 19 & Istmina & Chocó & --- & --- & --- & --- \\
\hline 20 & Juanchito & Valle del Cauca & --- & VII & $\mathrm{V}$ & V \\
\hline 21 & La Cumbre & Valle del Cauca & VIII & VII & VII & VII \\
\hline 22 & La Unión & Valle del Cauca & VII & VII & VI & VII \\
\hline 23 & Líbano & Tolima & IV & --- & --- & --- \\
\hline 24 & Manizales & Caldas & V & --- & V & V \\
\hline 25 & Medellín & Antioquia & IV & --- & IV & V \\
\hline 26 & Palmira & Valle del Cauca & V & VI & V & VI \\
\hline 27 & Pandi & Cundinamarca & IV & --- & $\mathrm{V}$ & V \\
\hline 28 & Pasto & Nariño & III & --- & --- & --- \\
\hline 29 & Pereira & Risaralda & VI & $\mathrm{V}$ & V & V \\
\hline 30 & Pichindé & Cali - Valle del Cauca & --- & ---- & ---- & $\mathrm{V}$ \\
\hline 31 & Piendamó & Cauca & VII & --- & --- & --- \\
\hline 32 & Popayán & Cauca & VI & VI & VI & VI \\
\hline 33 & Quibdó & Chocó & IV & --- & IV & V \\
\hline 34 & Restrepo & Valle del Cauca & VIII & VII & --- & VII \\
\hline 35 & Roldanillo & Valle del Cauca & VII & VI & VII & VI \\
\hline 36 & Salento & Quindío & IV & --- & $\mathrm{V}$ & V \\
\hline 37 & Sevilla & Valle del Cauca & --- & --- & VI & --- \\
\hline 38 & Subachoque & Cundinamarca & IV & --- & --- & --- \\
\hline 39 & Tadó & Chocó & --- & --- & --- & ---- \\
\hline 40 & Toro & Valle del Cauca & --- & ---- & ---- & VI \\
\hline 41 & Ubalá & Cundinamarca & IV & --- & --- & ---- \\
\hline 42 & Villahermosa & Tolima & IV & --- & --- & ---- \\
\hline 43 & Yotoco & Valle del Cauca & VIII & V & VI & V \\
\hline
\end{tabular}


La Figura 3 presenta, de manera gráfica, la comparación de la asignación de las intensidades macrosísmicas dada por los estudios anteriores en diversas poblaciones del territorio colombiano, conforme se discrimina en la Tabla 1.

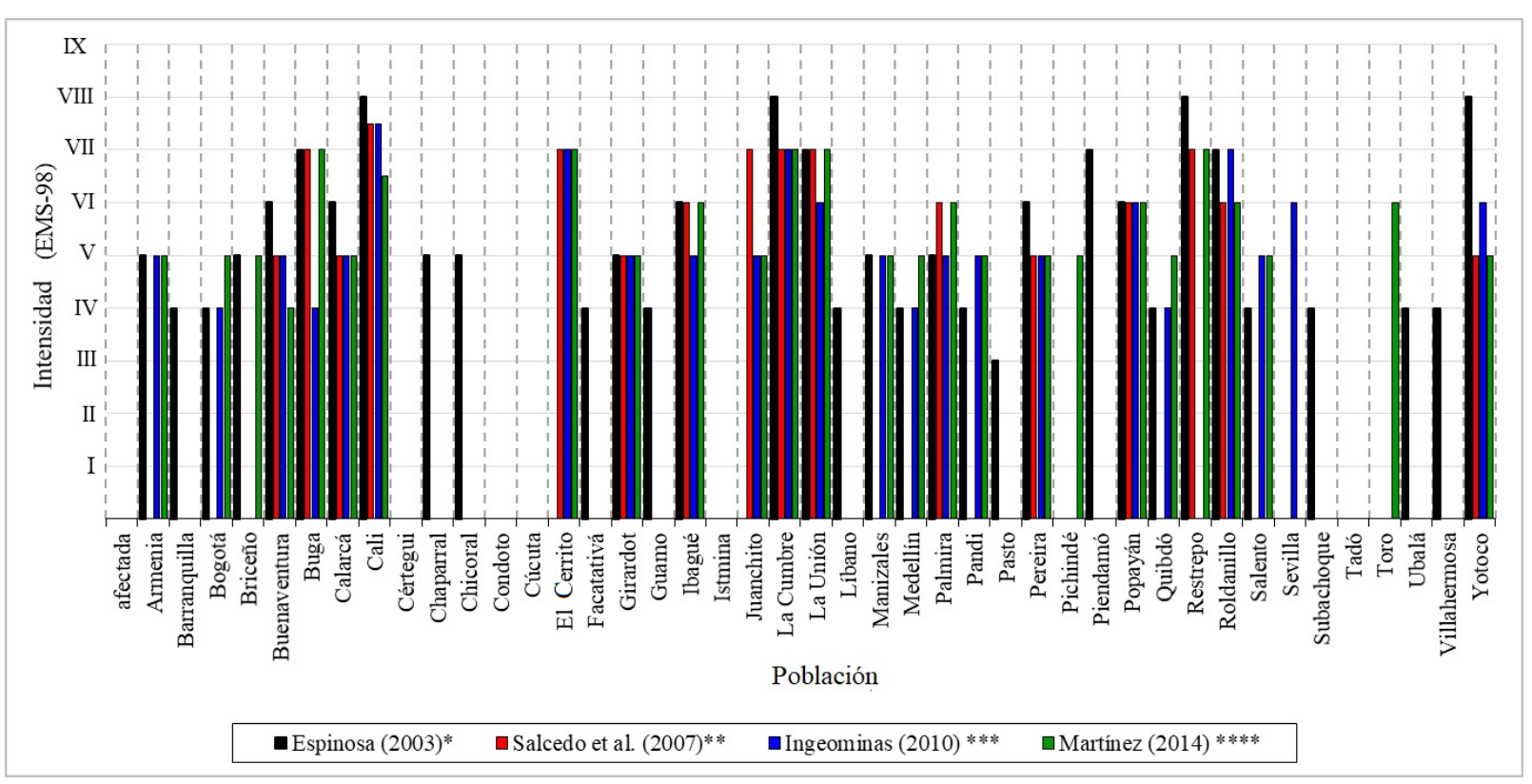

Figura 3. Intensidades macrosísmicas asignadas por estudios anteriores a diversas poblaciones afectadas por el terremoto del 07 de junio de 1925.

Para establecer la relación entre los diversos valores de intensidad asignados en los estudios anteriores, se aplicó el coeficiente de correlación lineal de Pearson, expresado como:

$$
r_{x y}=\frac{\sigma_{X Y}}{\sigma_{X} \sigma_{Y}}
$$

donde $\sigma_{X Y}$ es la covarianza muestral de las variables $(X$, $Y), \sigma_{X}$ es la desviación estándar muestral de la variable $X$ y $\sigma_{Y}$, la desviación estándar muestral de la variable $Y$.
El coeficiente de correlación es adimensional y varía en el intervalo $[-1,+1]$, donde -1 expresa una correlación máxima inversa, mientras +1 representa una correlación máxima directa; un valor de correlación 0 indica que no existe correlación alguna entre las variables consideradas. Este procedimiento ha sido usado por otros autores (Salcedo-Hurtado y Castaño-Castaño, 2011; Leal-Guzmán et al., 2018). La Tabla 2 muestra el resultado de la correlación entre las asignaciones de intensidad para el terremoto del 07 de junio de 1925, dadas por los estudios macrosísmicos anteriores.

Tabla 2. Coeficiente de correlación entre los diferentes valores de intensidad asignados en estudios anteriores para el terremoto del 07 de junio de 1925.

\begin{tabular}{lcccc}
\hline \multicolumn{1}{c}{ Fuentes de Información } & $\begin{array}{c}\text { Espinosa- } \\
\text { Baquero (2003) }\end{array}$ & $\begin{array}{c}\text { Salcedo-Hurtado } \\
\text { et al. (2007) }\end{array}$ & $\begin{array}{c}\text { INGEOMINAS } \\
\mathbf{( 2 0 1 0 )}\end{array}$ & $\begin{array}{c}\text { Martínez } \\
\mathbf{( 2 0 1 4 )}\end{array}$ \\
\hline Espinosa-Baquero (2003) & 1 & 0,485 & 0,388 & 0,459 \\
Salcedo-Hurtado et al. (2007) & 0,485 & 1 & 0,693 & 0,759 \\
INGEOMINAS (2010) & 0,388 & 0,693 & 1 & 0,816 \\
Martínez (2014) & 0,459 & 0,759 & 0,816 & 1 \\
\hline
\end{tabular}


Como se puede apreciar, independientemente del tipo de escala macrosísmica utilizada en cada estudio, se nota muy baja correlación entre los valores de intensidad asignados; la mayor correlación se presenta en las asignaciones dadas por INGEOMINAS (2010) y Martínez (2014), con un coeficiente del orden de 0,82 . La correlación que existe entre la evaluación hecha por Espinosa-Baquero (2003) con las demás fuentes es muy baja. El estudio de Espinosa-Baquero (2003) se toma como referencia debido a que es el que presenta la mayor cantidad de poblaciones evaluadas. Sin embargo, la evaluación de las intensidades en dicho estudio se realizó con base en la escala de Mercalli modificada, que, en los últimos años, en Colombia, ha entrado en desuso.

Así, la reevaluación de las intensidades macrosísmicas de este terremoto se hace necesaria, por un lado, debido a que los estudios macrosísmicos anteriores no unifican la información y tampoco realizan la evaluación de intensidades para todas las poblaciones afectadas. Por otro lado, por la disparidad o falta de correlación de las asignaciones anteriores, lo que muestra gran incertidumbre entre ellas, como se indica en la Tabla 2.

Estos aspectos marcan la necesidad de hacer nuevas interpretaciones de la información y reevaluación de la intensidad macrosísmica para el terremoto del 07 de junio de 1925, preferiblemente usando una única escala de intensidad, en este caso la EMS-98, cuya aplicación, como lo muestran Salcedo-Hurtado y Castaño-Castaño (2011), Salcedo-Hurtado y GómezCapera (2013) y Gómez-Capera et al. (2014), presenta ventajas frente a resultados obtenidos con otras escalas macrosísmicas tradicionalmente usadas en Colombia.

El presente estudio aporta nuevos datos macrosísmicos: por un lado, reporta daños y efectos ocasionados a varias poblaciones no señaladas en los estudios anteriores y, por el otro, propone nueva hipótesis de intensidad, en cada una de las poblaciones de diferentes departamentos afectadas por este terremoto, dejando puertas abiertas para que en estudios macrosísmicos posteriores se evalúen los parámetros sismológicos como el epicentro, la profundidad y la magnitud, entre otros (Blake, 1941; Shebalin, 1968, 1971; Bakun y Wentworth, 1997; Salcedo-Hurtado et al., 2021a).

\section{Metodología}

En el presente estudio se emplea el método intensivista (Rodríguez de la Torre, 1992), el cual consiste en ampliar el conocimiento de las particularidades de un evento sísmico histórico sobre el cual se tiene información de su ocurrencia en una fecha determinada, es decir, conociendo la existencia del evento se revisan las publicaciones periódicas, libros, documentos de archivos y otro tipo de información para obtener datos que contribuyan a analizar aspectos generales y específicos del evento, área de percepción y sus características (Rodríguez de la Torre, 1992, 1993; Salcedo-Hurtado y Tabares, 2001). Este proceso metodológico puede ser utilizado para el estudio de sismos ocurridos tanto en periodos preinstrumentales como recientes (Salcedo-Hurtado, 1999; SalcedoHurtado et al., 2007).

Cada evento sísmico, según sus características físicas, puede generar, en mayor o menor medida, daños y efectos que permiten la asignación de la intensidad macrosísmica, la cual puede variar en función de la escala utilizada. Para el caso del sismo del 07 de junio de 1925, diversos autores han propuesto valores de intensidad cuya calidad es bastante heterogénea debido, por un lado, a la escasa información recopilada, y, por el otro, al análisis realizado a la luz de las diversas escalas macrosísmicas usadas.

Sabiendo que existen diversos trabajos que presentan hipótesis de intensidades para del 07 de junio de 1925, y según Guidoboni y Stucchi (1993), se elige realizar una nueva investigación analizando y explotando los datos que están disponibles en las compilaciones sismológicas anteriores, mediante la ruta esquematizada en la Figura 4.

\section{Fuentes de información histórica}

En este estudio, la búsqueda de información se ha llevado a cabo en archivos, bibliotecas, hemerotecas, librerías, centros de documentación de diferentes ciudades. Acorde con la información contenida, los documentos obtenidos y revisados fueron categorizados como fuentes primarias y secundarias, principalmente.

Las fuentes primarias corresponden a documentos originales como libros, revistas, periódicos, diarios, documentos oficiales de instituciones públicas, informes técnicos y de investigación, que contienen información primaria o contemporánea con la fecha del evento y que narran los hechos observados por testigos directos. Por su parte, las fuentes secundarias hacen alusión a publicaciones como libros y artículos, que dan cuenta del evento como producto 
del análisis, reorganización de datos e información contenida en otros trabajos primarios originales.

\section{Captura y almacenamiento de la información}

Para capturar y almacenar la información se utilizó una ficha bibliográfica (Salcedo-Hurtado, 1999), donde se documentan los datos históricos sobre los efectos o daños ocasionados por el terremoto en cuestión. En esta ficha se hace la transcripción de la información encontrada en cada fuente bibliográfica. Esto se hace describiendo tanto la fuente donde se encontró la noticia como las características del evento sísmico, dentro de las cuales se detallan los daños o efectos tal como aparece en la noticia, sin modificar o corregir ningún apartado de la misma. Dado que la noticia sísmica aparece en diversas fuentes bibliográficas, se requiere sintetizar la información sobre los datos encontrados. Para ello, se elaboró una segunda ficha denominada "Ficha Resumen de Efectos del Terremoto" (FRET), en la cual se consignan los diferentes efectos con los cuales se evalúa la intensidad sísmica (SalcedoHurtado et al., 2007).

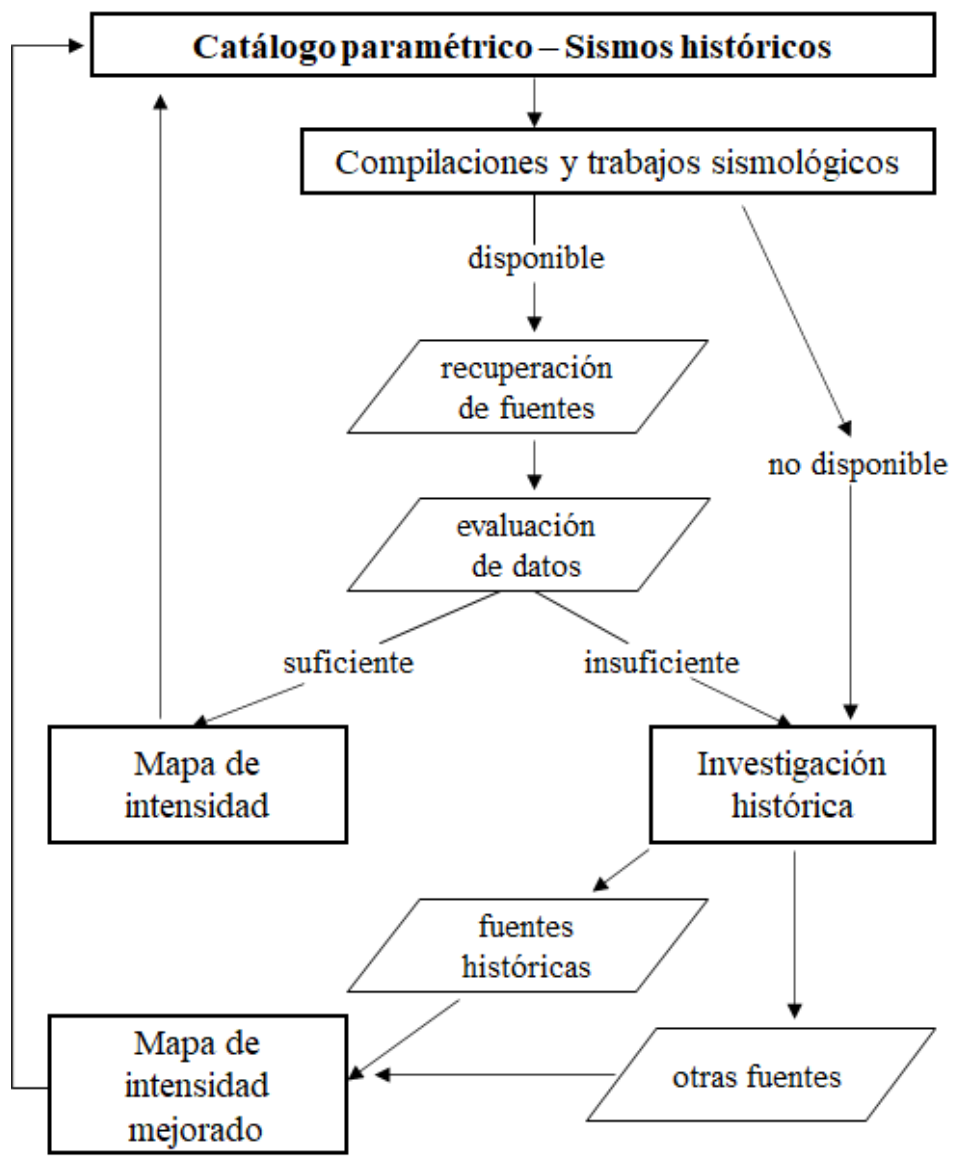

Figura 4. Esquema de procedimientos para la actualización del conjunto de datos macrosísmicos (modificado de Guidoboni y Stucchi, 1993).

\section{Asignación de intensidades}

La evaluación y asignación de intensidades se realiza mediante una escala macrosísmica que permita procesar la información sobre los efectos ocasionados por un terremoto. Para este estudio se empleó la Escala Macrosísmica Europea 1998 (EMS-98) que clasifica la intensidad del terremoto en 12 niveles, desde I (no sentido) hasta XII (Grünthal, 2009).
La valoración de intensidad realizada en el presente estudio introduce un factor de confiabilidad de la información y certeza en la hipótesis de la intensidad asignada, lo cual se hace mediante un factor de calidad Q, descrito como: A - el sismo fue sentido y la información es suficiente y veraz para dar un valor apropiado de intensidad; B - el sismo fue sentido, pero la información no es suficiente para dar un valor de 
intensidad confiable; $\mathrm{C}$ - la información es insuficiente y dudosa (Salcedo-Hurtado, 1999, 2002).

\section{Elaboración del mapa de intensidades e isosistas}

Una vez asignada la intensidad a cada uno de los lugares señalados por las diversas fuentes bibliográficas - noticias sísmicas encontradas sobre el evento bajo estudio donde se haya sentido el sismo o este haya causado algún tipo de efecto-, se procede a la elaboración del respectivo mapa de intensidades que indica el valor de intensidad asignada a cada población, acorde con la escala macrosísmica usada, en este caso EMS-98.

Las poblaciones afectadas han sido georreferenciadas acorde con la base cartográfica oficial para Colombia; con ello se realiza el trazado de las líneas de isosistas siguiendo una figura elíptica (Musson y Cecić, 2012) y empleando sistemas de información geográfica, de tal manera que cada población y su respectivo valor o punto de intensidad quede ubicado dentro de un área que represente un valor de intensidad, encerrada por su correspondiente isolínea.

Es posible que algún punto de intensidad quede ubicado fuera de su respectiva área de intensidad y aparezca dentro de una isosista que encierre otra área con valor de intensidad diferente; por ejemplo, un punto de intensidad $\mathrm{V}$ puede quedar ubicado dentro del área de una isosista de intensidad IV o VI, esto se debe a las variaciones inducidas por efectos locales y por el correcto trazado de las líneas de isosistas, que buscan guardar la forma de una elipse (Musson y Cecić, 2012). Es preciso aclarar que el espaciado de las isolíneas no necesariamente es homogéneo en todo el campo macrosísmico del respectivo terremoto.

\section{Resultados}

\section{Nueva investigación histórica}

Como resultado de los procesos metodológicos descritos anteriormente, la reevaluación de la intensidad macrosísmica del terremoto del $07 \mathrm{de}$ junio de 1925 permitió establecer los siguientes productos: en primer lugar, se clasificaron las fuentes bibliográficas encontradas que contienen información sobre el evento señalado; luego, se discriminaron los datos macrosísmicos descritos en cada fuente de información, clasificando los tipos y modos de daños y efectos causados en cada población afectada o en aquellas donde se reportó que el sismo al menos fue sentido; por último, se asignó o reevaluó el valor de intensidad en cada población, de acuerdo con la descripción de la Escala Macrosísmica Europea de 1998 (Grünthal, 2009), y se elaboraron los mapas de intensidad e isosistas.

\section{Clasificación de las fuentes de información}

Fuentes primarias:

Se trata de información obtenida de los archivos, se caracteriza por ser escritos o relatos de testigos sobre los efectos del terremoto. En este sentido, se realizó la visita al Archivo General de la Nación, en la ciudad de Bogotá; al Archivo Central del Cauca, en la ciudad de Popayán; al Archivo Histórico Municipal de Cali, en la ciudad de Cali, y a los Archivos Históricos de Cartago y Buga, en los municipios de Cartago y Buga, respectivamente. En Bogotá, se realizaron visitas, a su vez, a la Academia Colombiana de Ciencias Naturales, Físicas y Exactas, en la ciudad de Bogotá y a la Hemeroteca Nacional. También se realizaron búsquedas, aunque no siempre fructíferas en la consecución de noticias relacionadas con este evento, en la Biblioteca Luis Ángel Arango; Biblioteca del Banco de la República, en las ciudades de Cali y Popayán, Biblioteca de la Universidad del Valle y Centro de Documentación del Departamento de Historia de la Universidad del Valle, en la ciudad de Cali. El Anexo 1 presenta el listado de las fuentes primarias consultadas (información proveniente de libros, periódicos y revistas) para el presente estudio.

\section{Fuentes secundarias:}

Las fuentes secundarias son aquellas publicaciones no contemporáneas con la fecha de ocurrencia del evento que, además de reproducir datos sobre los efectos causados en algunas poblaciones, pueden proporcionar información adicional para la asignación de intensidades o sobre parámetros como la magnitud, profundidad y localización epicentral. En el Anexo 2 se relacionan las fuentes secundarias consultadas, que en este caso corresponden a informes técnicos de instituciones públicas y artículos publicados en revistas especializadas.

\section{Discriminación de daños y efectos causados por el terremoto}

A partir de la información extraída de cada una de las fuentes, se discriminan los daños y efectos en cada población mencionada, los cuales, según la 
escala EMS-98, se categorizan desde débiles hasta totalmente devastadores, en cuanto afecten personas, objetos, construcciones y el entorno natural (efectos sismogeológicos). Estos últimos corresponden a efectos complejos influenciados con frecuencia por distintos factores como la estabilidad inherente de una ladera, el nivel freático, etc. (Grünthal, 2009).

Principales efectos en personas, objetos y construcciones: fueron diversos los efectos reportados por los habitantes de las poblaciones donde el sismo del 07 de junio de 1925 fue sentido o produjo daños. La información al respecto fue extraída de las diferentes fuentes bibliográficas descritas anteriormente. Es importante tener en cuenta que para la descripción de los efectos se hizo la transcripción exacta de cómo la población percibió y relató el acontecimiento, esto con el fin de realizar la asignación de la intensidad con la Escala Macrosísmica Europea (EMS-98). Los efectos en objetos se manifiestan en el sentido de que diversos elementos observados por las personas, se balancean, caen y, en muchos casos, se rompen a causa de la vibración sísmica. Al igual que los efectos en personas, para la determinación de intensidades, es necesario mostrar la información puntual de los efectos en objetos ocasionados por el evento sísmico. Por último, se tiene que las afectaciones en construcciones pueden impactar seriamente en el estado y la calidad de las viviendas, que pueden llegar a sufrir colapso parcial o incluso total de las mismas. Esto provocaría más consecuencias e incremento de la intensidad macrosísmica. En el Anexo 3 se muestra, de manera resumida, los efectos principales causados en las personas, objetos y construcciones en algunas de las poblaciones donde las diversas fuentes consultadas indican algún tipo de afectación provocada por el terremoto del 07 de junio de 1925. Se nota que los mayores efectos fueron reportados en la ciudad de Cali.

Efectos en el entorno natural: a pesar de que el evento sísmico del 07 de junio de 1925 ocasionó varios daños en la ciudad de Cali, no hubo reportes de efectos en el medio ambiente, tales como deslizamientos o eyección de lodo de manera súbita; por el contrario, el comportamiento de los suelos aparentemente fue estable, a excepción de la población de Piendamó en el departamento del Cauca, donde se reportaron efectos de este tipo (Tabla 3 ).

Tabla 3. Sismo 07 de junio de 1925. Efectos en el entorno natural.

Población afectada

Piendamó
Efectos en el entorno natural

Se abrió una grieta que mide 180 varas de largo, 40 centímetros de ancho por lo mismo de profundidad.

\section{Reevaluación de intensidades macrosísmicas}

De acuerdo con la información recopilada y la respectiva clasificación y discriminación de los efectos y daños ocasionados, se reevaluó la intensidad macrosísmica en cada una de las poblaciones donde el sismo del 07 de junio de 1925 fue sentido o dejó algún tipo de afectación; la Tabla 4 muestra el resultado de este proceso.

En la Figura 5 se presenta un diagrama de barras que indica el nivel de intensidad asignada a cada una de las poblaciones donde se evaluó la respectiva información macrosísmica. En esta figura se aprecia que, en relación con los estudios macrosísmicos anteriores sobre el terremoto del 07 de junio de 1925 (ver Figura 3 ), el presente trabajo complementa la información asignando la intensidad a cada una de las poblaciones afectadas según la información de las diferentes fuentes bibliográficas que se consultaron.
Los valores de intensidad presentados en la Tabla 4 y la Figura 5 muestran que a la ciudad de Santiago de Cali se le ha asignado el mayor nivel de intensidad, clasificada con daños severos (VIII), debido a que se dieron afectaciones serias e incluso colapsos parciales en muchas de las edificaciones presentes para la época del evento, lo que causó pánico extremo entre los habitantes de la ciudad.

Obsérvese que la información utilizada, aunque para algunas poblaciones puede ser poca, ha permitido dar asignaciones de intensidad de buena calidad, a pesar de que algunos valores pueden ser intermedios, como es el caso de las intensidades asignadas a los municipios de La Cumbre y La Unión en el departamento del Valle del Cauca, donde se reportan valores de VI-VII y V-VI, respectivamente. Debe quedar claro que estos no corresponden a valores decimales de 6,5 y 5,5 , esto significa que la información reportada, por ejemplo, para el caso del municipio de La Cumbre, contiene 
algunos elementos que permiten asignar intensidad son suficientes para que este sea el valor final de la de VI, pero además indica datos del nivel VII que no intensidad en dicha población.

Tabla 4. Asignación de intensidades para el terremoto del 07 de junio de 1925 en Colombia.

\begin{tabular}{|c|c|c|c|c|c|c|}
\hline No. & $\begin{array}{c}\text { Población } \\
\text { afectada }\end{array}$ & Departamento & $\begin{array}{c}\text { Latitud } \\
{ }^{\circ} \mathbf{N}\end{array}$ & $\begin{array}{c}\text { Longitud } \\
{ }^{\circ} \mathbf{O}\end{array}$ & $\begin{array}{c}\text { Intensidad } \\
\text { EMS-98 }\end{array}$ & $\begin{array}{c}\text { Calidad } \\
\text { (Factor Q)* }\end{array}$ \\
\hline 1 & Armenia & Quindío & 4,54 & 75,67 & IV & $\mathrm{B}$ \\
\hline 2 & Barranquilla & Atlántico & 10,99 & 74,82 & III & B \\
\hline 3 & Bogotá & Cundinamarca & 4,66 & 74,07 & IV & $\mathrm{B}$ \\
\hline 4 & Briceño & Boyacá & 5,69 & 73,92 & IV & B \\
\hline 5 & Buenaventura & Valle del Cauca & 3,87 & 77,00 & VI & B \\
\hline 6 & Buga & Valle del Cauca & 3,89 & 76,29 & VI & B \\
\hline 7 & Calarcá & Quindío & 4,53 & 75,64 & VI & A \\
\hline 8 & Cali & Valle del Cauca & 3,43 & 76,50 & VIII & A \\
\hline 9 & Cerrito & Valle del Cauca & 5,37 & 76,61 & VI & B \\
\hline 10 & Cértegui & Chocó & 3,72 & 75,48 & III & B \\
\hline 11 & Chaparral & Tolima & 4,21 & 74,98 & VI & B \\
\hline 12 & Chicoral & Tolima & 5,09 & 76,65 & IV & B \\
\hline 13 & Condoto & Chocó & 7,89 & 72,51 & III & B \\
\hline 14 & Cúcuta & Norte de Santander & 3,68 & 76,31 & II & B \\
\hline 15 & Facatativá & Cundinamarca & 4,80 & 74,35 & IV & B \\
\hline 16 & Girardot & Cundinamarca & 4,30 & 74,80 & IV & B \\
\hline 17 & Guamo & Tolima & 4,03 & 74,96 & IV & B \\
\hline 18 & Ibagué & Tolima & 4,43 & 75,20 & VI & B \\
\hline 19 & Istmina & Chocó & 5,15 & 76.68 & IV & B \\
\hline 20 & Juanchito & Valle del Cauca & 3,45 & 76,47 & VII & B \\
\hline 21 & La Cumbre & Valle del Cauca & 3,64 & 76,56 & VI-VII & B \\
\hline 22 & La Unión & Valle del Cauca & 4,53 & 76,10 & V-VI & B \\
\hline 23 & Líbano & Tolima & 4,92 & 75,06 & IV & B \\
\hline 24 & Manizales & Caldas & 5,06 & 75,48 & V & B \\
\hline 25 & Medellín & Antioquia & 6,25 & 75,56 & III & B \\
\hline 26 & Palmira & Valle del Cauca & 3,53 & 76,29 & V-VI & B \\
\hline 27 & Pandi & Cundinamarca & 4,19 & 74,48 & IV & B \\
\hline 28 & Pasto & Nariño & 1,21 & 77,27 & IV & B \\
\hline 29 & Pereira & Risaralda & 4,80 & 75,69 & V & B \\
\hline 30 & Piendamó & Cauca & 2,64 & 76,53 & V & B \\
\hline 31 & Popayán & Cauca & 2,46 & 76,58 & VI & B \\
\hline 32 & Quibdó & Chocó & 5,68 & 76,65 & IV & B \\
\hline 33 & Restrepo & Valle del Cauca & 3,82 & 76,52 & VI & B \\
\hline 34 & Roldanillo & Valle del Cauca & 4,41 & 76,15 & VI & B \\
\hline 35 & Salento & Quindío & 4,63 & 75,57 & IV & B \\
\hline 36 & Sevilla & Valle del Cauca & 4,26 & 75,93 & VII & B \\
\hline 37 & Subachoque & Cundinamarca & 4,92 & 74,17 & III-IV & B \\
\hline 38 & Tadó & Chocó & 5,26 & 76,56 & III & B \\
\hline 39 & Toro & Valle del Cauca & 4,63 & 76,08 & $\mathrm{~V}$ & B \\
\hline 40 & Ubalá & Cundinamarca & 4,74 & 73,53 & III-IV & B \\
\hline 41 & Villahermosa & Tolima & 5,03 & 75,12 & III-IV & B \\
\hline 42 & Yotoco & Valle del Cauca & 3,86 & 76,38 & V-VI & B \\
\hline
\end{tabular}

* Factor $Q: A$ - el sismo fue sentido y la información es suficiente y veraz; $B$ - el sismo fue sentido, pero la información no es suficiente; $C$ - la información es insuficiente y dudosa. 


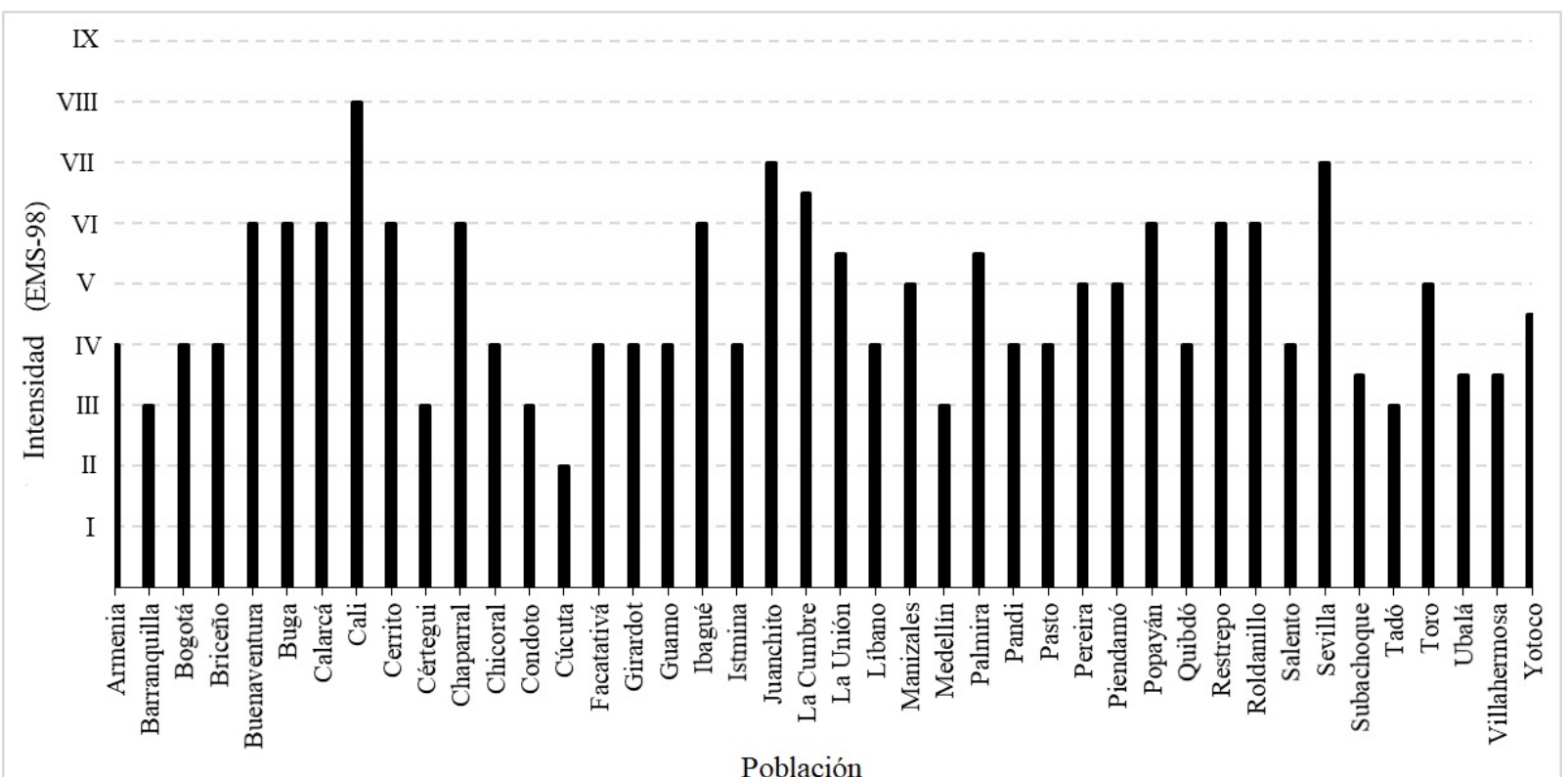

Figura 5. Histograma de asignación de intensidades macrosísmicas en diversas poblaciones afectadas por el terremoto del 07 de junio de 1925 en el suroccidente colombiano.

\section{Mapa de intensidad mejorado}

En la Figura 6 se presenta el mapa de puntos de intensidad macrosísmica, donde se distribuyen las intensidades asignadas, en la escala EMS-98, a cada una de las 42 poblaciones que, de acuerdo con los datos reportados por las diferentes fuentes de información, han sido afectadas por dicho evento sísmico (ver Tabla 4). Puede observarse que estos puntos geográficamente se distribuyen principalmente en los departamentos del Valle del Cauca, Tolima, Cundinamarca, Chocó, Quindío y Cauca; también se tienen puntos de intensidad en los departamentos de Risaralda, Caldas, Antioquia y Boyacá. El Valle del Cauca es el departamento con mayor número de poblaciones afectadas, específicamente 13 municipios con reportes de algún tipo de afectación.

La distribución geográfica de estos puntos de intensidad marca una ligera tendencia SSO-NNE, donde los mayores valores de intensidad se asignan a las poblaciones del departamento del Valle del Cauca, con intensidades desde V hasta VIII, y el valor máximo se asigna a la ciudad de Cali, a partir de la cual se aprecia el decaimiento de la intensidad tanto hacia el NNE como hacia el SSO, marcando mayor elongación hacia el NNE. Los puntos de menor intensidad mostrados en la Tabla 4, asignados a las ciudades de Barranquilla y Cúcuta con valores de II y III, respectivamente, por razones de la escala del mapa, no se muestran en la Figura 6.

\section{Mapa de isosistas y epicentro macrosísmico}

Las isosistas, en esencia, son la representación gráfica del campo macrosísmico de un terremoto dado a partir de la irradiación de la energía sísmica desde el foco. En el presente trabajo se trazan las curvas de isosistas correspondientes al campo macrosísmico del terremoto del 07 de junio de 1925 ocurrido en el suroccidente colombiano (Figura 7). En este mapa se nota que la isolínea de mayor valor corresponde a la zona afectada con intensidad VIII, que encierra la ciudad de Santiago de Cali; las demás isolíneas varían en intensidades entre IV y VII en la escala EMS-98. Las isolíneas dibujadas tienen forma elíptica mostrando que su eje mayor tiene una tendencia SSO-NNE, dirección en la cual se supone la mejor propagación de la energía elástica. Mientras que el eje menor aparece en la dirección ONO-ESE, en la cual se tiene mayor atenuación de la energía elástica.

En este caso, se evalúa el epicentro macrosísmico del terremoto del 07 de junio de 1925, el cual preliminarmente se ha ubicado en las coordenadas: $3,45^{\circ}$ norte y $76,49^{\circ}$ oeste, en cercanías de la ciudad de Cali, donde se evaluó la intensidad máxima. 


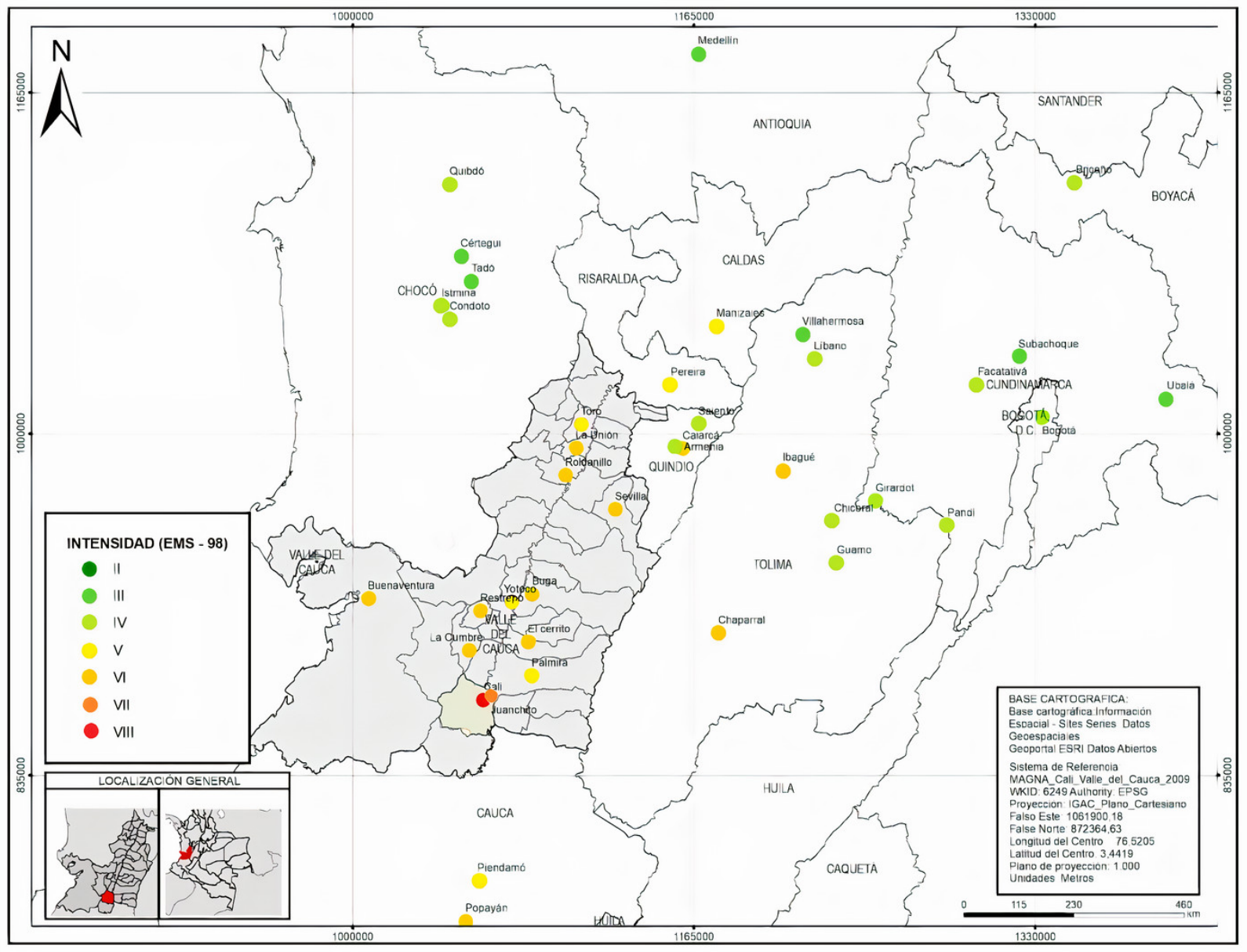

Figura 6. Mapa de centros de intensidad macrosísmica del terremoto del 07 de junio de 1925.

Varios autores y metodologías hacen uso de los mapas de isosistas para evaluar diversos parámetros macrosísmicos de los terremotos, como es el caso de leyes de atenuación, profundidad focal, magnitud, entre otros (Barbano et al., 1979; Bottari et al., 1982a, 1982b, 1986; Shebalin, 1971, 1974, 1991; Grases y Rodríguez, 2001; Marín-Arias et al., 2006, 2009; Salcedo-Hurtado et al., 2021a).

\section{Discusión}

Es claro que para lograr un mejor conocimiento de los terremotos se deben realizar estudios instrumentales y macrosísmicos complementarios que conjuntamente conduzcan a precisar las características y los parámetros físicos (Salcedo-Hurtado et al., 2021a), así como las particularidades del foco sísmico, la propagación de la energía sísmica y la distribución de las intensidades a partir de los daños y efectos causados en la superficie terrestre. Sin embrago, para el caso de terremotos históricos o eventos recientes ocurridos en zonas donde es notoria la baja densidad de instrumentación sísmica, una buena opción de análisis la constituyen los estudios macrosísmicos (Salcedo et al., 2021b). En esta dirección, en el territorio colombiano se han realizado diversos trabajos donde exitosamente se aplican técnicas y métodos de análisis macrosísmico para calcular y precisar los parámetros sísmicos de algunos terremotos históricos y recientes (GómezCapera y Salcedo-Hurtado, 2000; Marín-Arias et al., 2006, 2009; Salcedo-Hurtado y Castaño-Castaño, 2011; Salcedo-Hurtado y Gómez-Capera, 2013; Gómez-Capera et al., 2014; Salcedo-Hurtado et al., 2021a). Así mismo, se ha hecho el ejercicio para un terremoto histórico ocurrido en territorio venezolano (Salcedo et al., 2021b).

Acerca del terremoto del 07 de junio de 1925, ocurrido en la región del suroccidente colombiano, se han realizado algunos trabajos macrosísmicos como el de Espinosa-Baquero (2003), Salcedo-Hurtado et al. 
(2007), INGEOMINAS (2005a, 2010) y Martínez (2014). Cada uno de ellos presenta su hipótesis de intensidades, pero no todos cubren información en todas las poblaciones que, de acuerdo con las fuentes de información, se mencionan como afectadas por este terremoto. Además, las asignaciones de intensidad se hacen en escalas diferentes y se presenta gran incertidumbre en cuanto a las valoraciones mismas.

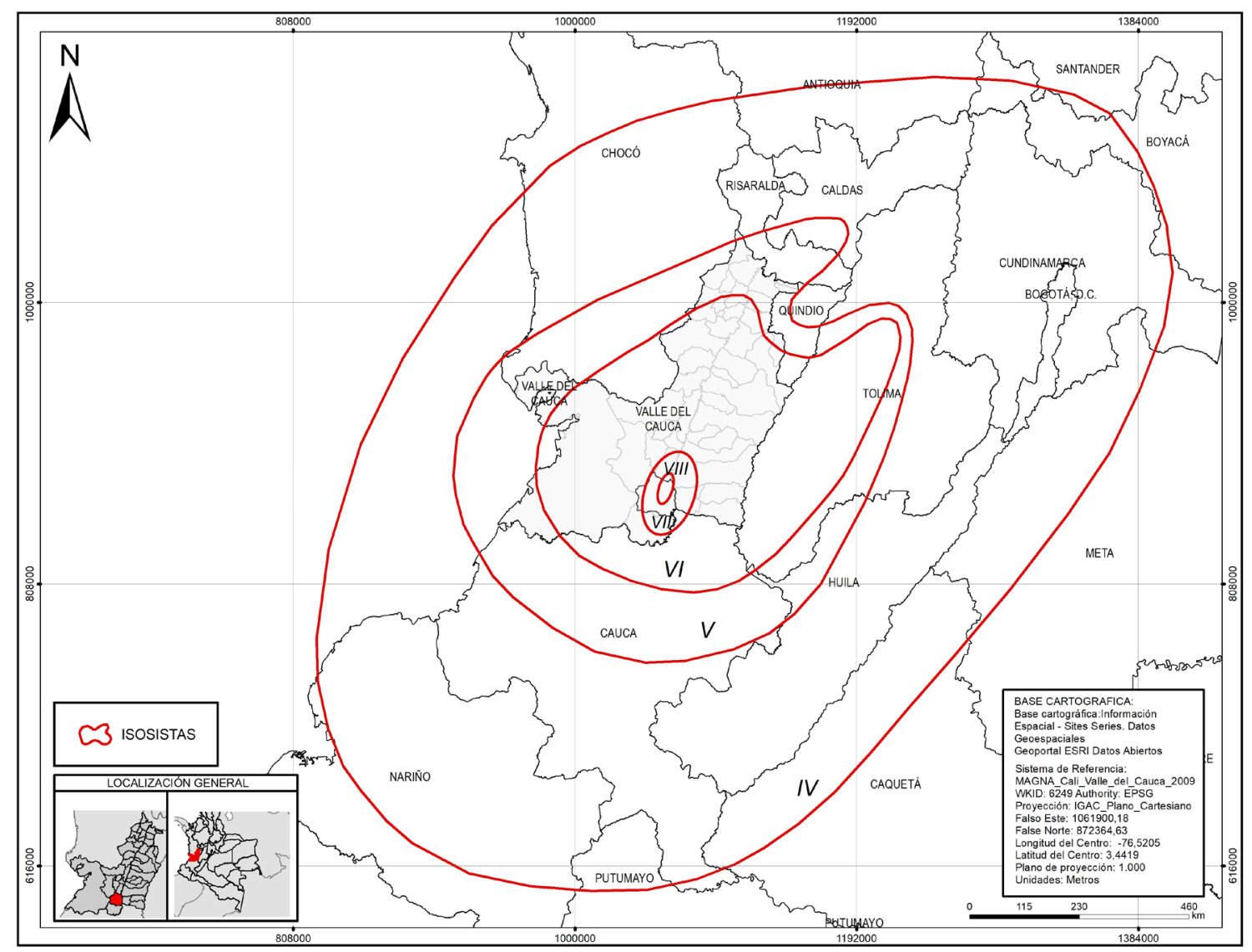

Figura 7. Mapa de isosistas del terremoto sísmico del 07 de junio de 1925.

En el presente trabajo se hace una nueva investigación histórica sobre este terremoto y se muestran las disimilitudes en el uso de las escalas macrosísmicas y la baja correspondencia entre los valores de intensidad macrosísmica asignados. De esta manera, se realiza la reevaluación de la intensidad macrosísmica en cada una de las poblaciones afectadas usando una escala moderna, reduciendo la incertidumbre con los valores asignados en estudios anteriores. Así, se ha logrado ampliar el área de percepción y precisar las asignaciones de intensidades, permitiendo elaborar un mapa de intensidad más denso, ya que se encontraron nueve (9) puntos de intensidad adicionales con respecto al estudio realizado por Espinosa-Baquero (2003), lo que significa que para el presente estudio se registraron 42 poblaciones afectadas mientras que el estudio de Espinosa-Baquero reportó 33 poblaciones con algún tipo de daño (Tabla 5).
El epicentro macrosísmico, preliminarmente definido en este trabajo, ha sido ubicado en las coordenadas $3,45^{\circ}$ de latitud norte y $76,49^{\circ}$ de longitud oeste, cerca de la ciudad de Cali, y se asocia con el sistema de falla Cali-Patía (París y Romero, 1994; Nivia, 2001), que, como lo muestra la Figura 1, representa el límite oriental de la secuencia del Cretáceo superior de la cordillera Occidental y controla además el límite occidental del valle aluvial del río Cauca (Nivia, 2001). En la Figura 8 se muestra una relación entre la intensidad y la distancia al epicentro de cada una de las poblaciones afectadas por el terremoto sísmico del 07 de junio de 1925, según las asignaciones del presente estudio.

Es preciso señalar que en el presente estudio no se notan puntos de intensidad con grandes anomalías, donde se evidencie un fuerte incremento de la intensidad en relación 
con la distancia al epicentro (Tabla 5 y Figura 8). En este sentido, solo llama la atención el caso del municipio de Sevilla en el departamento del Valle del Cauca, que siendo una población ubicada a unos $106 \mathrm{~km}$ al noreste del epicentro muestra un valor de intensidad de VII, similar a poblaciones cercanas al epicentro, lo que puede estar relacionado con el hecho de que este municipio se localiza en el flanco occidental de la cordillera Central, donde aflora una faja amplia de rocas basálticas que constituyen geoformas de origen denudacional que alcanzan alturas hasta de $10 \mathrm{~m}$, y sus suelos están predominantemente formados por cenizas volcánicas (CVC-Universidad del Valle, 2011). En Colombia este tipo de suelos son susceptibles a inestabilidad, erosión y agrietamiento dependiendo de las condiciones climáticas y de la cobertura vegetal (Lizcan et al., 2006).

Tabla 5. Intensidades sísmicas evaluadas en el presente estudio y las propuestas por Espinosa-Baquero (2003) en las poblaciones afectadas por el terremoto del 07 de junio de 1925.

\begin{tabular}{|c|c|c|c|c|c|}
\hline No. & $\begin{array}{c}\text { Nombre de la } \\
\text { población afectada }\end{array}$ & Departamento & $\begin{array}{c}\text { I (MM) } \\
\text { Espinosa-Baquero } \\
(2003) \\
\end{array}$ & $\begin{array}{c}\text { I (EMS-98) } \\
\text { Presente estudio }\end{array}$ & $\Delta(\mathbf{k m})$ \\
\hline 1 & Armenia & Quindío & $\mathrm{V}$ & IV & 144,33 \\
\hline 2 & Barranquilla & Atlántico & IV & III & 857,53 \\
\hline 3 & Bogotá & Cundinamarca & IV & IV & 273,6 \\
\hline 4 & Briceño & Boyacá & V & IV & 378,71 \\
\hline 5 & Buenaventura & Valle del Cauca & VI & VI & 67,87 \\
\hline 6 & Buga & Valle del Cauca & VII & VI & 32,07 \\
\hline 7 & Calarcá & Quindío & VI & VI & 145,97 \\
\hline 8 & Cali & Valle del Cauca & VIII & VIII & 11,1 \\
\hline 9 & Cértegui & Chocó & --- & III-IV & 215,69 \\
\hline 10 & Chaparral & Tolima & $\mathrm{V}$ & VI & 105,19 \\
\hline 11 & Chicoral & Tolima & $\mathrm{V}$ & IV & 194,27 \\
\hline 12 & Condoto & Chocó & --- & $\mathrm{V}$ & 180,64 \\
\hline 13 & Cúcuta & Norte de Santander & --- & II & 681,79 \\
\hline 14 & El Cerrito & Valle del Cauca & --- & VI & 40,04 \\
\hline 15 & Facatativá & Cundinamarca & IV & IV & 284,56 \\
\hline 16 & Girardot & Cundinamarca & $\mathrm{V}$ & IV & 210,56 \\
\hline 17 & Guamo & Tolima & IV & IV & 181,84 \\
\hline 18 & Ibagué & Tolima & VI & VI & 176,83 \\
\hline 19 & Istmina & Chocó & --- & IV & 149,5 \\
\hline 20 & Juanchito & Valle del Cauca & --- & VII & 14,06 \\
\hline 21 & La Cumbre & Valle del Cauca & VIII & VI-VII & 29,34 \\
\hline 22 & La Unión & Valle del Cauca & VII & V-VI & 128,38 \\
\hline 23 & Líbano & Tolima & IV & IV & 225,44 \\
\hline 24 & Manizales & Caldas & $\mathrm{V}$ & V & 211,86 \\
\hline 25 & Medellín & Antioquia & IV & III & 326,34 \\
\hline 26 & Palmira & Valle del Cauca & V & V-VI & 31,5 \\
\hline 27 & Pandi & Cundinamarca & IV & IV & 238,42 \\
\hline 28 & Pasto & Nariño & III & IV & 274,97 \\
\hline 29 & Pereira & Risaralda & VI & $\mathrm{V}$ & 171,37 \\
\hline 30 & Piendamó & Cauca & VII & V & 82,23 \\
\hline 31 & Popayán & Cauca & VI & VI & 109,39 \\
\hline 32 & Quibdó & Chocó & IV & IV & 277,5 \\
\hline 33 & Restrepo & Valle del Cauca & VIII & VI & 39,96 \\
\hline 34 & Roldanillo & Valle del Cauca & VII & VI & 116,13 \\
\hline 35 & Salento & Quindío & IV & IV & 167,35 \\
\hline 36 & Sevilla & Valle del Cauca & --- & VII & 106,29 \\
\hline 37 & Subachoque & Cundinamarca & IV & III & 309,14 \\
\hline 38 & Tadó & Chocó & -- & III-IV & 202,76 \\
\hline
\end{tabular}




\section{Continuación Tabla 5.}

\begin{tabular}{cccccc}
\hline No. & $\begin{array}{c}\text { Nombre de la } \\
\text { población afectada }\end{array}$ & Departamento & $\begin{array}{c}\text { I (MM) } \\
\text { Espinosa-Baquero } \\
(\mathbf{2 0 0 3})\end{array}$ & $\begin{array}{c}\text { I (EMS-98) } \\
\text { Presente estudio }\end{array}$ & $\Delta(\mathbf{k m})$ \\
\hline 39 & Toro & Valle del Cauca & --- & V & 141,34 \\
40 & Ubalá & Cundinamarca & IV & III-IV & 371,59 \\
41 & Villahermosa & Tolima & IV & III-IV & 224,34 \\
42 & Yotoco & Valle del Cauca & VIII & V-VI & 51,69 \\
\hline
\end{tabular}

$\Delta(\mathrm{km})$, distancia al epicentro.

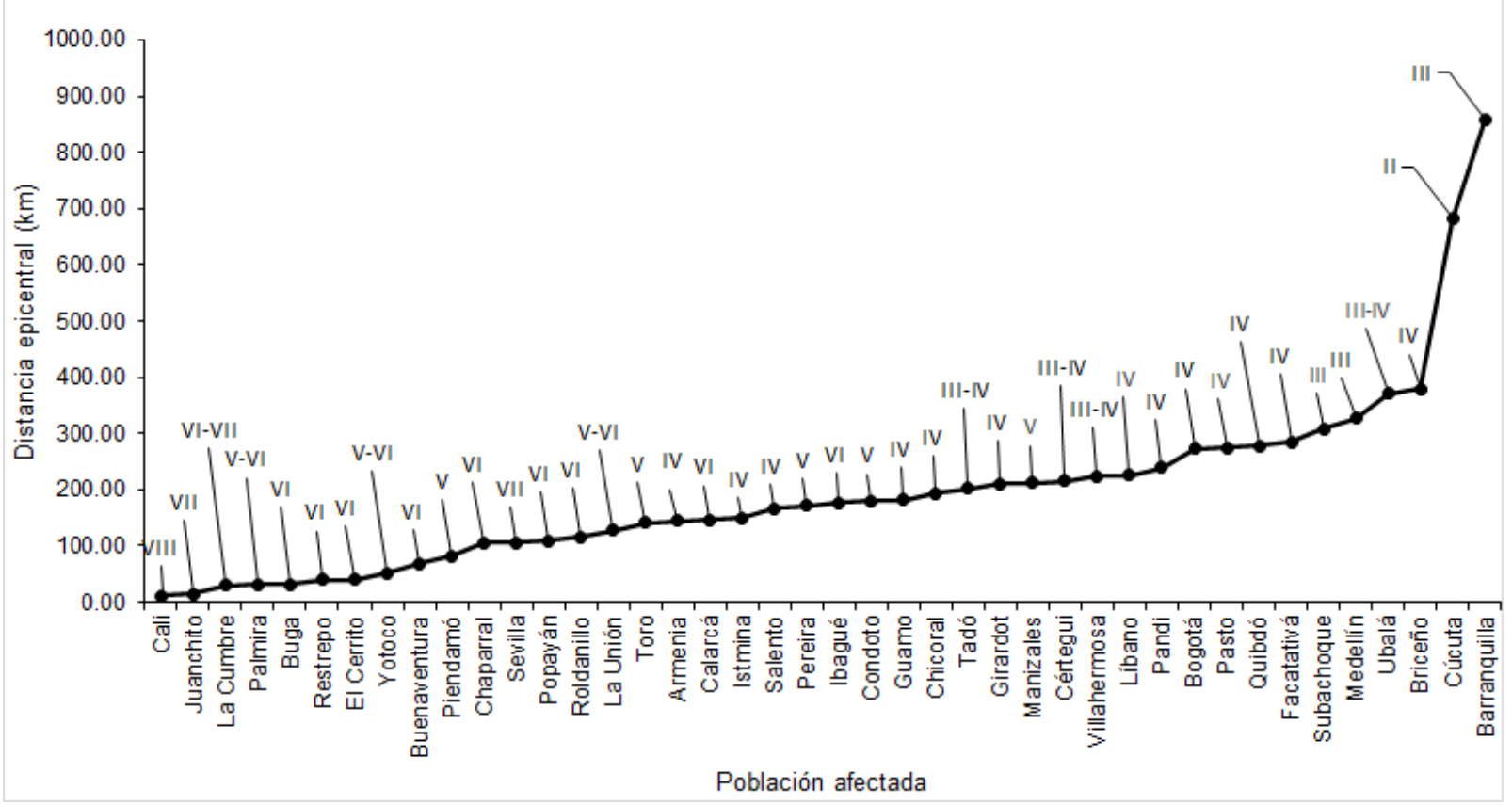

Figura 8. Relación intensidad-distancia en las poblaciones afectadas por el terremoto sísmico del 07 de junio de 1925 , según las asignaciones del presente estudio.

En este estudio se ha construido un mapa de isosistas, que no había sido trazado en estudios anteriores debido a la escaza cantidad de puntos de intensidad. Se espera que este resultado permita la aplicación de otros métodos macrosísmicos empíricos apropiados para establecer un modelo de atenuación de la intensidad acorde con las estructuras tectónicas presentes; así mismo, este trabajo abre la puerta para realizar otros estudios macrosísmicos que conduzcan a determinar los parámetros focales aplicando técnicas y métodos usados exitosamente en otras regiones (Barbano et al., 1979; Bottari et al., 1982a, 1982b, 1986; Shebalin, 1971, 1974, 1991; Grases y Rodríguez, 2001; MarínArias et al., 2006, 2009; Salcedo et al., 2021b), con el fin de alimentar el catálogo paramétrico regional y nacional que, a su vez, mejorarán el conocimiento para la evaluación de la amenaza, la vulnerabilidad y el riesgo sísmico de la región.

\section{Conclusiones}

Por los efectos y daños causados a diversas poblaciones de la región suroccidental del territorio colombiano, el terremoto ocurrido el 07 de junio de 1925 puede considerarse como uno de los eventos que posiblemente contribuye al incremento de la amenaza y el riesgo sísmico de la región del suroccidente colombiano. Por tales circunstancias, el estudio de las particularidades de este sismo ha sido tema de interés para diferentes autores, quienes han aportado datos e información de tipo instrumental y macrosísmico para lograr un mejor conocimiento de sus características físicas.

Desde el punto de vista instrumental, varios estudios muestran resultados que, al variar entre ellos los principales parámetros sismológicos del evento, dejan cierta incertidumbre en cuanto a la magnitud, 
localización y profundidad del foco. Por el lado de los datos macrosísmicos, diversos autores aportan información sobre los efectos y daños causados en poblaciones, especialmente del departamento del Valle del Cauca, asignándoles valores de intensidad en diversas escalas macrosísmicas. Las hipótesis de intensidad dadas en los estudios macrosísmicos anteriores mantienen baja correlación estadística entre ellos, lo cual implica el grado de incertidumbre en las evaluaciones realizadas.

Para el presente trabajo, se ha realizado una nueva investigación histórica, incluyendo otras fuentes bibliográficas obtenidas en archivos, hemerotecas y bibliotecas de diversas zonas del país, logrando con ello la obtención de nuevos datos que, con relación a los estudios macrosísmicos anteriores, amplían el número de poblaciones donde se reporta algún tipo de daño o afectación, permitiendo densificar la información para, por un lado, cubrir el "gap" geográfico que presentan los estudios anteriores y, por el otro, lograr mejor asignación de las intensidades. Para la revaloración de la intensidad se ha utilizado la Escala Macrosísmica Europea EMS98, que ha sido usada exitosamente en otros trabajos que evalúan intensidades de sismos ocurridos en el territorio colombiano. En total se presentan 42 puntos de intensidad en diferentes poblaciones, lo cual permite tener un mapa de intensidad más denso que el obtenido en estudios previos.

Dicho mapa de intensidades muestra que la distribución geográfica de los puntos de intensidad marca una ligera tendencia SSO-NNE, donde los mayores valores se asignan a las poblaciones ubicadas en el departamento del Valle del Cauca, en un rango de intensidad que va desde V hasta VIII, cuyo valor máximo se reporta en la ciudad de Cali. A partir de aquí, se nota el decaimiento de la intensidad tanto hacia el NNE como hacia el SSO, marcando mayor elongación hacia el NNE, situación que se marca claramente en el trazado de las curvas de isosistas obtenido a partir de este estudio. Con el mapa de isosistas se logró proponer el epicentro macrosísmico, que preliminarmente estaría ubicado en las coordenadas $3,45^{\circ}$ norte y $76,49^{\circ}$ oeste.

El presente trabajo aporta datos para que en el futuro se realicen nuevos estudios que apliquen técnicas y métodos macrosísmicos apropiados para evaluar de manera más precisa los parámetros sismológicos del terremoto del 07 de junio de 1925, especialmente la magnitud y la profundidad focal, y con ello mejorar la información de los catálogos sismológicos regional y nacional del territorio colombiano.

\section{Agradecimientos}

Este trabajo fue realizado en el marco del proyecto "Análisis del escenario de riesgo sísmico en Santiago de Cali ante la ocurrencia de un terremoto similar al de junio de 1925", financiado y registrado por la Vicerrectoría de Investigaciones de la Universidad del Valle, con el código CI 4354. El éxito en la búsqueda de información no hubiese sido posible sin el apoyo de los directivos y funcionarios de la Biblioteca Luis Ángel Arango; el Archivo Histórico Nacional, en la ciudad de Bogotá; el Archivo Histórico Central del Cauca, en Popayán; el Archivo Histórico de Cali; la Biblioteca Departamental de Cali; el Centro Documental Banco de la República; la Biblioteca Mario Carvajal de la Universidad del Valle, en la ciudad de Cali, quienes nos brindaron su invaluable colaboración. Expresamos nuestro agradecimiento a los miembros del Grupo de Investigación Georiesgos, que con su esfuerzo y colaboración hicieron posible este trabajo. Asimismo, expresamos nuestro profundo agradecimiento a los doctores Franck A. Audemard M. de la Universidad Central de Venezuela y Gustavo Barrantes Castillo de la Universidad Nacional de Costa Rica, por sus valiosos comentarios, observaciones y correcciones que fueron fundamentales para mejorar la calidad y contenido de este trabajo.

\section{Referencias}

Audemard, F.A.; Ollarves, R.; Bechtold, M.; Díaz, G.; Beck, C.; Carrillo, E.; Pantosti, D.; Diederix, H. (2008). Trench investigation on the main strand of the Boconó fault in its central section, at Mesa del Caballo, Mérida Andes, Venezuela. Tectonophysics, 459(1-4), 38-53. https://doi. org/10.1016/j.tecto.2007.08.020

Audemard, F.A. (1998). Contribución de la paleosismología a la sismicidad histórica: los terremotos de 1610 y de 1894 en los Andes venezolanos meridionales. Revista Geográfica Venezolana, 39(1-2), 87-105.

Audemard, F.A. (2003). Estudios paleosísmicos por trincheras en Venezuela: métodos, alcances, aplicaciones, limitaciones y perspectivas. Revista Geográfica Venezolana, 44(1), 11-46.

Audemard, F.A. (2005). Paleoseismology in Venezuela: objectives, methods, applications, limitations and 
perspectives. Tectonophysics, 408(1-4), 29-61. https://doi.org/10.1016/j.tecto.2005.05.034

Bakun, W.H.; Wentworth, C.M. (1997). Estimating earthquake locations and magnitude from seismic intensity data. Bulletin of the Seismological Society of America, 87(6), 1502-1521.

Barbano, M.S.; Bottari, A.; Carvani, P.; Cosentino, M.; Federico, B.; Fonte, G.; Lo Giudice, E.; Lombardo, G.; Patane, G. (1979). Macroseismic Study of the Gulf of Patti Earthquake in the Geostructural Frame of North - Eastern Sicily. Bollettino - Societa Geologica Italiana, 98(2), 155-174.

Blake, A. (1941). On the estimation of focal depth from macroseismic data. Bulletin of the Seismological Society of America, 31(3), 225-231. https://doi. org/10.1785/BSSA0310030225

Bolt, B.A. (2003). Earthquakes. 5th edition. W.H. Freeman \& Company.

Bottari, A.; Federico, B.; Lo Giudice, E. (1982a). Nuove evidenze di correlazione tra l'attenuazione macrosísmica ed i campi di lineamienti strutturali nell'Italia meridionale. Annals of Geophysics, 35(1), 5-26. https://doi.org/10.4401/ag-4669

Bottari, A.; Giovani, L.; Lo Giudice, E.; Longo, V.; Spadea, M.; Vecchi, M. (1982b). Regional Macroseismic Field of the 1980 Irpinia Earthquake. Annals of Geophysics, 35(1), 27-84. https://doi.org/10.4401/ag-4670

Bottari, A.; Carapezza, E.; Carapezza, M.; Carvani, P.; Cefali, E.; Lo Giudice, E.; Pandolfo, C. (1986). The 1908 Messina Strait earthquake in the regional geostructural framework. Journal of Geodynamics, 5(3-4), 275-302. https://doi. org/10.1016/0264-3707(86)90010-4

Castrillón-Arboleda, D. (1986). Historia de los terremotos de Popayán. En: Muros de Papel (pp. 23-35). Universidad del Cauca.

CERESIS. (1985). Catálogo de terremotos para América del Sur-Colombia. Datos de hipocentros e intensidades. Vol. 4.

CVC. (2019). Manual del usuario. Visor geográfico avanzado.
CVC-Universidad del Valle. (2011). Estudio de amenaza, vulnerabilidad, escenarios de riesgo y diseño de ingeniería y tratamientos de mitigación ante movimientos en masa en los barrios Monserrate y Cafetero del municipio de Sevilla, Valle del Cauca. Resumen general. Contrato Interadministrativo 0252 .

Engdahl, E.R.; Villaseñor, A. (2002). Global Seismicity: 1900-1999. In: W.H.K. Lee, H. Kanamori, P.C. Jennings, C. Kisslinger (eds.). International Geophysics (pp. 665-690). Vol. 81, Part A, Chapter 41, Academic Press. https://doi. org/10.1016/S0074-6142(02)80244-3

Espinosa-Baquero, A. (2003). Historicidad sísmica de Colombia. 07/06/1925 - Isla Gorgona. INGEOMINAS.

GERSCO. (1987). Informe de sismicidad histórica. Grupo de Estudio de Riesgo Sísmico del Suroccidente Colombiano. Inédito.

Gouin, P. (1994). Interpreting original reports on historical earthquakes in Quebec. In: P. Albini, A. Moroni (eds.). Materials of the CEC Project "Review of Historical Seismicity in Europe" (pp. 191-194). CNR - Istituto di Ricerca sul Rischio Sismico.

Gómez-Capera, A.A.; Salcedo-Hurtado, E. de J. (2000). Determinación de la profundidad focal de algunos sismos ocurridos en Colombia, a partir de datos macrosísmicos. Revista de la Red Sismológica Regional del Eje Cafetero, 5, 12-16.

Gómez-Capera, A.A.; Salcedo-Hurtado, E. de J.; Bindi, D.; Choy, J.E.; García-Peláez, J. (2014). Localización y magnitud del terremoto de 1785 en Colombia calculadas a partir de intensidades macrosísmicas. Revista de la Academia Colombiana de Ciencias Exactas, Físicas $y$ Naturales, 38(147), 206-217. https://doi. org/10.18257/raccefyn.63

Grases, J.; Rodríguez, J.A. (2001). Estimaciones de magnitud de sismos venezolanos a partir de mapas de isosistas. II Congreso Venezolano de Ingeniería Sísmica, Mérida, Venezuela.

Grünthal, G. (2009). Escala Macrosísmica Europea 1998. Conseil de l'Europe, Cahiers du Centre 
Européen de Géodynamique et de Séismologie. Edición española F. Lorenzo Martín.

Guidoboni, E.; Stucchi, M. (1993). The contribution of historical records of earthquakes to the evaluation of seismic hazard. Annals of Geophysics, 36(3-4), 201-215. https://doi.org/10.4401/ag-4264

Gutenberg, B.; Richter, C. (1954). Seismicity of the Earth and Associated Phenomena. Prinnceton University.

INGEOMINAS. (2005a). Estudio de microzonificación sísmica de Cali: Informe No.1-4. Estudio de Sismicidad Histórica Regional.

INGEOMINAS. (2005b). Estudio de microzonificación sísmica de Cali: Informe No.1-3. Investigaciones Neotectónicas en el Centro Occidente Colombiano.

INGEOMINAS. (2010). Estudio macrosísmico del sismo del 07 de junio de 1925, Cali (Valle del Cauca). Instituto Colombiano de Geología y Minería. Bogotá.

ISC-GEM. (2015). V 2.0 of the ISC-GEM Catalogue.

Izquierdo-Álvarez, A. (1999). Intensidad macrosísmica. Física de la Tierra, 11, 237-252.

Lay, T.; Wallace, T.C. (1995). Modern Global Seismology. Academic Press.

Leal-Guzmán, A; Vásquez-Stanescu, R.; Rodríguez, J.A.; Audemard, F.A. (2018). Reevaluación del terremoto del 29 de octubre de 1900 en Venezuela. Primera parte: estimación de intensidades. GEOMINAS, 46(77), 139-154.

Lizcan, A.; Herrera, M.C.; Santamarina, J.C. (2006). Suelos derivados de cenizas volcánicas en Colombia. Revista Internacional de Desastres Naturales, Accidentes e Infraestructura Civil, 6(2), 167-197.

Marín-Arias, J.P.; Castillo-González, H.; SalcedoHurtado, E. de J. (2006). Modelo geométrico del foco del terremoto de Popayán (Colombia) a partir de datos macrosísmicos. Boletín de Geología, 28(2), 93-109.
Marín-Arias, J.P.; Valencia-Muñoz, L.Y.; RendónCardona, L.M. (2009). Análisis macrosísmico del terremoto de Murindó - Antioquia (Colombia) octubre de 1992. Boletín de Geología, 31(1), 85-93.

Martínez, M. (2014). Sismicidad histórica de la región del Valle del Cauca y zonas aledañas. Tesis, Universidad del Valle, Colombia.

Mendoza, D.; Rosales, C.; Velásquez, A.; Meyer, H. (2004). Revisión macrosísmica de los parámetros hipocentrales del terremoto del 7 de Junio de $1925(\mathrm{Ms}=6,8)$ en Colombia. I Congreso Latinoamericano de Sismología, II Congreso Colombiano de Sismología. Armenia, Colombia.

Meyer, H. (1990). Proyecto integral para la mitigación del riesgo sísmico en Cali. Seminario Desastres Sísmicos en Grandes Ciudades, Santiago de Cali, Colombia.

Musson, R.M.; Cecić, I. (2012). Intensity and Intensity Scales. In: P. Bormann (ed.). New Manual of Seismological Observatory Practice 2 (NMSOP-2) (pp. 1-41). Deutsches GeoForschungsZentrum GFZ. https://doi.org/10.2312/GFZ.NMSOP-2 $\operatorname{ch} 12$

Nivia, J.A. (2001). Mapa Geológico Departamento del Valle del Cauca Escala 1:250.000, Memoria Explicativa.

París, G.; Romero, J. (1994). Fallas activas en Colombia. Boletín Geológico, 34(2-3), 4-25.

París, G.; Machette, M.; Dart, R.; Haller, K. (2000). Map of Database of Quaternary Faults and Folds in Colombia and its Offshore Regions. USGS. Open-File Report 00-0284.

Ramírez, J.E. (1975). Historia de los Terremotos en Colombia. 2da. ed. Instituto Geográfico Agustín Codazzi.

Rodríguez de la Torre, F. (1992). La geografía y la historia de los sismos. Geocrítica, 16(97), 1-72.

Rodríguez de la Torre, F. (1993). Lecturas sistemáticas de prensa periódica. Hacia una revisión de la sismicidad europea durante los siglos XVII y XVIII. In: P. Albini, A. Moroni (eds.). Materials of 
the CEC Project "Review of Historical Seismicity in Europe" (pp. 247-258). CNR - Istituto di Ricerca sul Rischio Sismico.

Rodríguez, J.A.; Audemard, F.A. (2003). Sobrestimaciones y limitaciones en los estudios de sismicidad histórica con base en casos venezolanos. Revista Geográfica Venezolana, 44(1), 47-75.

Salcedo-Hurtado, E. de J. (1999). Estudio de sismicidad histórica en la región de Bucaramanga (Colombia). Revista de la Academia Colombiana de Ciencias Exactas, Físicas y Naturales, 23(87), 233-248.

Salcedo-Hurtado, E. de J.; Tabares, L.M. (2001). Sismicidad histórica una herramienta de trabajo en macrosísmica. VIII Congreso Colombiano de Geología. Manizales, Colombia.

Salcedo-Hurtado, E. de J. (2002). Sismicidad histórica y análisis macrosísmico de Bucaramanga. Boletín Geológico, 40(1), 1-180.

Salcedo-Hurtado, E. de J.; Romero-Vergara, M.D.; Vallejo-Chocué, M.A. (2007). Contribución al análisis macrosísmico del terremoto del 7 de junio de 1925: principales efectos en la ciudad de Cali. Revista de la Academia Colombiana de Ciencias Exactas, Físicas y Naturales, 31(120), 379-394.

Salcedo-Hurtado, E. de J.; Castaño-Castaño, A.N. (2011). Reevaluación macrosísmica del terremoto del 12 de Julio de 1785 en Colombia. Boletín de Geología, 33(2), 15-32.

Salcedo-Hurtado, E. de J.; Gómez-Capera, A.A. (2013). Estudio macrosísmico del terremoto del 18 de octubre de 1743 en la región central de Colombia. Boletín de Geología, 35(1), 109-128.

Salcedo-Hurtado, E. de J.; Audemard, F.A.; GarcíaMillán, N. (2021a). Parámetros focales del terremoto del 28 de abril de 1894 en los Andes venezolanos usando datos macrosísmicos. Revista de la Academia Colombiana de Ciencias Exactas, Físicas y Naturales, 45(175), 591-606. https:// doi.org/10.18257/raccefyn. 1195

Salcedo-Hurtado, E. de J.; Gómez-Capera, A.A.; Mora-Páez, H. (2021b). Análisis complementario entre parámetros instrumentales y macrosísmicos: terremoto de mayo 24, 2008 en Quetame, Colombia. Boletín de Geología, 43(2), 163-184. https://doi.org/10.18273/revbol.v43n2-2021009

Shebalin, N.V. (1968). Método de utilización de datos ingeniero-geológicos en la zonación sísmica. En: Zonificación sísmica de la URSS (pp. 95-111). Moscú: Nauka. (En ruso).

Shebalin, N.V. (1971). Cálculo del Tamaño y Posición del Foco del Terremoto de Tashkent Mediante Datos Macrosísmicos e Instrumentales. En: El terremoto de Tashkent de 1966. (pp. 68-80). Tashkent (En ruso).

Shebalin, N.V. (1974). Focos de Terremotos Fuertes en el Territorio de la URSS. Nauka: 52. (En ruso).

Shebalin, N.V. (1991). Cálculo del Tamaño y Posición del Foco del Terremoto de Spitak Mediante Datos Macrosísmicos. En: Problemas de la Ingeniería Sísmica (pp. 35-44). Nauka. (En ruso).

Stucchi, M. (1994). Recommendation for the compilation of a European parametric earthquake catalogue, with special reference to historical records. In: P. Albini, A. Moroni (eds.). Materials of the CEC Project "Review of historical seismicity in Europe" (pp. 181-190). CNR - Istituto di Ricerca sul Rischio Sismico.

\section{Archivo}

Delgado, P. (1925). Creen en un terremoto en Colombia, gran sensación en Nueva York por temblor.10, junio, 1925. p. 2.

Hernández, G. (s.f.). La iglesia mayor. La historia de Santiago de Cali. Archivo Histórico de Cali. 286p.

\section{Hemerografía}

ABC. Quindío. 09, junio. (1925). El domingo a las 7 menos 10 minutos de la noche, se sintió un fuerte temblor en esta ciudad, p. 1.

CORREO DEL CAUCA. Cali. 08, junio. (1925). Cali al influjo de un terremoto, la catástrofe sísmica de anoche, el fatídico domingo 7. 
EL GRÁFICO. Bogotá. 10, junio. (1925). El fuerte temblor del domingo, Resumen noticioso de la semana. $\mathrm{N}^{\circ}$ 738. p. 1.

EL ESPECTADOR. Bogotá. 08, junio. (1925). Daños materiales y desgracias personales, las predicciones D'Bendandi. El temblor de ayer en la república y en el exterior. p. 1.

EL RELATOR. Cali. 08, junio. (1925). El pavoroso terremoto de anoche. $1,2 \mathrm{p}$.
EL TIEMPO. Bogotá. 08, junio. (1925). El movimiento causó grandes daños en Cali - Ayer se sintieron fortísimos temblores. 1,3,4 p.

MUNDO AL DÍA. Bogotá. 08, junio. (1925). El epicentro en el Quindío - Gran terror en el Valle por el temblor de ayer. 1 y $11 \mathrm{p}$.

Fecha de recibido: 25 de julio de 2020

Fecha de aprobado: 23 de agosto de 2021 
Anexo 1. Fuentes primarias respecto a la búsqueda de información sobe el sismo del 07 de junio de 1925 en el suroccidente colombiano.

\begin{tabular}{|c|c|c|c|c|c|}
\hline Nombre/Título & Autor & Año & $\begin{array}{c}\text { Tipología de la } \\
\text { fuente }\end{array}$ & Páginas & Localización \\
\hline $\begin{array}{l}\text { Historia de los } \\
\text { terremotos en Popayán. } \\
\text { En: Muros de Papel }\end{array}$ & $\begin{array}{l}\text { Diego Castrillón- } \\
\text { Arboleda }\end{array}$ & 1986 & Libro & $23-35$ & $\begin{array}{l}\text { Archivo } \\
\text { Histórico de } \\
\text { Popayán - } \\
\text { Cauca } \\
\end{array}$ \\
\hline $\begin{array}{c}\text { La historia de Cali/La } \\
\text { iglesia Mayor }\end{array}$ & $\begin{array}{l}\text { Guillermo } \\
\text { Hernández }\end{array}$ & s.f & Libro & 21 & $\begin{array}{c}\text { Archivo } \\
\text { Histórico de Cali }\end{array}$ \\
\hline $\begin{array}{l}\text { El epicentro en el } \\
\text { Quindío }\end{array}$ & Mundo al día & 1925 & Periódico & 1 y 11 & $\begin{array}{l}\text { Hemeroteca } \\
\text { Biblioteca } \\
\text { Nacional } \\
\end{array}$ \\
\hline $\begin{array}{l}\text { Creen en un terremoto } \\
\text { en Colombia }\end{array}$ & Puyo Delgado & 1925 & Periódico & 2 & $\begin{array}{l}\text { Hemeroteca } \\
\text { Biblioteca } \\
\text { Nacional }\end{array}$ \\
\hline $\begin{array}{l}\text { Resumen noticioso de } \\
\text { la semana/El fuerte } \\
\text { temblor del domingo }\end{array}$ & El Gráfico & 1925 & Revista & 1 & $\begin{array}{l}\text { Hemeroteca } \\
\text { Biblioteca } \\
\text { Nacional }\end{array}$ \\
\hline $\begin{array}{l}\text { El temblor de ayer en la } \\
\text { República }\end{array}$ & El Espectador & 1925 & Periódico & 1 & $\begin{array}{l}\text { Hemeroteca } \\
\text { Biblioteca } \\
\text { Nacional }\end{array}$ \\
\hline $\begin{array}{l}\text { Ayer se sintieron } \\
\text { fortísimos temblores }\end{array}$ & El Tiempo & 1925 & Periódico & $1,3,4$ & $\begin{array}{c}\text { Hemeroteca } \\
\text { Biblioteca } \\
\text { Nacional } \\
\end{array}$ \\
\hline $\begin{array}{c}\text { El domingo a las } 7 \\
\text { menos } 10 \text { minutos de } \\
\text { la noche, se sintió un } \\
\text { fuerte temblor en esta } \\
\text { ciudad }\end{array}$ & $\mathrm{ABC}$ & 1925 & Periódico & 1 & $\begin{array}{l}\text { Hemeroteca } \\
\text { Biblioteca } \\
\text { Nacional }\end{array}$ \\
\hline $\begin{array}{l}\text { Cali al influjo de un } \\
\text { terremoto/La catástrofe } \\
\text { sísmica de anoche }\end{array}$ & Correo del Cauca & 1925 & Periódico & 7 & $\begin{array}{c}\text { Hemeroteca } \\
\text { Biblioteca } \\
\text { Nacional } \\
\end{array}$ \\
\hline $\begin{array}{c}\text { El pavoroso terremoto } \\
\text { de anoche }\end{array}$ & El Relator & 1925 & Periódico & 1,2 & $\begin{array}{l}\text { Hemeroteca } \\
\text { Biblioteca } \\
\text { Nacional }\end{array}$ \\
\hline
\end{tabular}

Anexo 2. Fuentes secundarias respecto a la búsqueda de información sobre el sismo del 07 de junio de 1925 en el suroccidente colombiano.

\begin{tabular}{|c|c|c|c|c|c|}
\hline Nombre o Título & Autor & Año & $\begin{array}{c}\text { Tipología de la } \\
\text { Fuente }\end{array}$ & Páginas & Localización \\
\hline M 6.3 - Colombia & USGS-GEM & s.f & $\begin{array}{c}\text { Documento en } \\
\text { línea }\end{array}$ & $\cdots$ & Archivo web \\
\hline 07/06/1925 - Isla Gorgona & $\begin{array}{l}\text { Armando Espinosa- } \\
\text { Baquero }\end{array}$ & 2003 & $\mathrm{CD}$ & $\ldots$ & A.C.N.F y E (A) \\
\hline $\begin{array}{l}\text { Sismicidad histórica de la } \\
\text { región del Valle del Cauca } \\
\text { y zonas aledañas }\end{array}$ & Maryluz Martínez & 2014 & Informe & 172 & $\begin{array}{l}\text { Documento } \\
\text { electrónico }\end{array}$ \\
\hline
\end{tabular}




\section{Continuación Anexo 2.}

\begin{tabular}{|c|c|c|c|c|c|}
\hline Nombre o Título & Autor & Año & $\begin{array}{c}\text { Tipología de la } \\
\text { Fuente }\end{array}$ & Páginas & Localización \\
\hline $\begin{array}{l}\text { Contribución al análisis } \\
\text { macrosísmico del sismo del } \\
07 \text { de junio de } 1925\end{array}$ & Salcedo-Hurtado et al. & 2007 & Informe & 16 & $\begin{array}{l}\text { Documento } \\
\text { electrónico }\end{array}$ \\
\hline $\begin{array}{l}\text { Microzonificación sísmica } \\
\text { de Cali - Informe histórico }\end{array}$ & INGEOMINAS & $2005 a$ & Informe & $33-37$ & $\begin{array}{l}\text { Documento } \\
\text { electrónico }\end{array}$ \\
\hline $\begin{array}{l}\text { Estudio macrosísmico del } \\
\text { sismo del } 07 \text { de junio de } \\
1925\end{array}$ & INGEOMINAS & 2010 & Informe & & $\begin{array}{l}\text { Documento } \\
\text { electrónico }\end{array}$ \\
\hline
\end{tabular}

Anexo 3. Principales efectos en las personas, objetos y construcciones causados por el sismo del 07 de junio de 1925 , reportados en algunas de las poblaciones indicadas por las diversas fuentes bibliográficas.

\begin{tabular}{|c|c|c|c|}
\hline \multirow{2}{*}{$\begin{array}{l}\text { Población } \\
\text { afectada }\end{array}$} & \multicolumn{3}{|c|}{ Tipo de efecto } \\
\hline & En personas & En objetos & En construcciones \\
\hline Cali & $\begin{array}{l}\text { Fue fatal para Cali, el temblor fue } \\
\text { extremadamente fuerte. Murió un } \\
\text { muchacho a quien lo mató una enorme } \\
\text { piedra del Salón Moderno. Se dice } \\
\text { que hay numerosos heridos por causa } \\
\text { de los desprendimientos de techos } \\
\text { en varios edificios, la oscuridad y un } \\
\text { aguacero que principió a caer en el } \\
\text { momento en que se sintió el temblor, } \\
\text { contribuyeron a aumentar el pánico, } \\
\text { por todas partes se escuchaban } \\
\text { gritos de angustia. Resultó herido de } \\
\text { gravedad Antonio López, han salido } \\
\text { de la ciudad muchas familias, y, otras } \\
\text { preparan viaje apresuradamente. } \\
\text { La ciudad en sus habitaciones, ha } \\
\text { quedado seriamente lesionada. El } \\
\text { pánico cundió extraordinariamente } \\
\text { en la ciudad, pasaron la noche fuera } \\
\text { de sus casas, en carpas improvisadas, } \\
\text { temiendo nuevos derrumbes. El } \\
\text { espanto era indecible. }\end{array}$ & $\begin{array}{l}\text { El reloj de la iglesia de } \\
\text { San Pedro se detuvo en } \\
\text { la hora precisa en que } \\
\text { se efectuó el terremoto. } \\
\text { La botica se perdió } \\
\text { totalmente, pues los } \\
\text { frascos se quebraron. En } \\
\text { casi todas las cantinas } \\
\text { se registran daños de } \\
\text { consideración, pues se } \\
\text { quebraron casi todas } \\
\text { las botellas. La oficina } \\
\text { telegráfica de Cali quedó } \\
\text { completamente aislada, } \\
\text { porque las baterías se } \\
\text { rompieron. Los teléfonos } \\
\text { se cruzaron y muchas } \\
\text { cuerdas se reventaron. }\end{array}$ & $\begin{array}{l}\text { Ocasionó numerosos y valiosos daños } \\
\text { en la ciudad; como la destrucción de la } \\
\text { iglesia de la Ermita, el terremoto afectó } \\
\text { la cúpula de la iglesia de San Francisco. } \\
\text { La ciudad estuvo a punto de perecer } \\
\text { bajo la tierra y de quedar convertida } \\
\text { en un montón de trágicos escombros. } \\
\text { Muchísimas de las casas se cuartearon, el } \\
\text { temblor quebrantó seriamente el templo } \\
\text { de San Pedro su fachada y campanario, } \\
\text { San Francisco, La Ermita, San Nicolás } \\
\text { (las torres en construcción del templo de } \\
\text { San Nicolás se agrietaron desde el suelo } \\
\text { hasta lo alto del edificio), La Merced, } \\
\text { y la torre de Santa Librada. Además. } \\
\text { Varios edificios particulares sufrieron } \\
\text { graves daños. En el Hotel Francia se } \\
\text { desplomaron las paredes interiores, se } \\
\text { vino íntegramente al suelo, en varios } \\
\text { edificios se desplomaron bastantes } \\
\text { techos. Sufrieron graves averías las } \\
\text { casas de don Miguel Calero y de la } \\
\text { familia Vélez; el edificio Lora. Puede } \\
\text { decirse que, en Cali, no ha quedado } \\
\text { ninguna casa sin avería, de más o menos } \\
\text { consideración. }\end{array}$ \\
\hline Calarcá & $\begin{array}{l}\text { Fue de una intensidad violentísima. } \\
\text { Hasta ahora se sabe que a consecuencia } \\
\text { de él hubo dos muertos. Las gentes } \\
\text { son presa del mayor pánico, todas } \\
\text { las casas fueron abandonadas por sus } \\
\text { ocupantes. El terror es incontenible. }\end{array}$ & $\begin{array}{l}\text { Se cayeron los postes } \\
\text { que sostienen las cuerdas } \\
\text { de la luz. }\end{array}$ & $\begin{array}{l}\text { A consecuencia de un derrumbe de } \\
\text { una pared habían quedado muertas } \\
\text { dos personas, las torres de la iglesia } \\
\text { se vinieron abajo, ocasionó algunos } \\
\text { daños y desgracias, causó daños en las } \\
\text { habitaciones. }\end{array}$ \\
\hline
\end{tabular}




\section{Continuación Anexo 3.}

\begin{tabular}{|c|c|c|c|}
\hline \multirow{2}{*}{$\begin{array}{c}\text { Población } \\
\text { afectada }\end{array}$} & \multicolumn{3}{|c|}{ Tipo de efecto } \\
\hline & En personas & En objetos & En construcciones \\
\hline La Cumbre & $\begin{array}{l}\text { Ocasionó muerte instantánea a doña } \\
\text { Joaquina A. Reina consternación. La } \\
\text { tranquilidad de los habitantes se tornó } \\
\text { en locura. En la desesperación, unos } \\
\text { gritaban, otros lloraban desconsolados } \\
\text { más fue necesario recogerlos del } \\
\text { suelo desmayados. Fueron momentos } \\
\text { de alarma y de dolor. El señor don } \\
\text { Rogerio González, dueño del Hotel } \\
\text { Tuller, en estado de completa locura } \\
\text { nerviosa, se arrojó por una de las } \\
\text { tribunas del edificio y que van hacia } \\
\text { la calle. Esta caída le ocasionó la } \\
\text { dislocación del pie derecho, la señorita } \\
\text { Dioselina Paredes sufrió un accidente } \\
\text { bastante grave. }\end{array}$ & 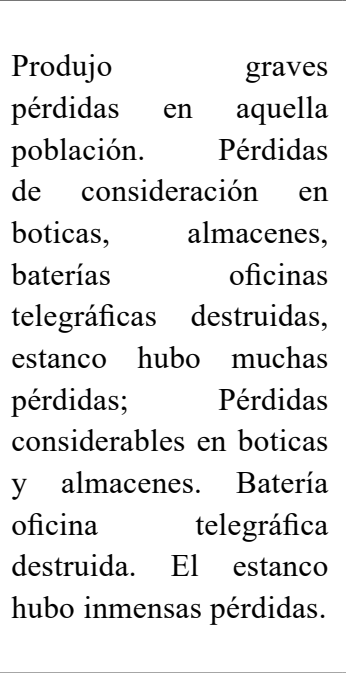 & $\begin{array}{l}\text { Bodega del Ferrocarril desplomose en } \\
\text { parte. }\end{array}$ \\
\hline Piendamó & El pueblo aterrado & $\begin{array}{l}\text { Fue una noche de } \\
\text { espanto; todos los relojes } \\
\text { se pararon, los autos } \\
\text { perdieron el control. } \\
\text { Destruyó baterías } \\
\text { de algunas oficinas, } \\
\text { ocasionó enormes daños } \\
\text { materiales. }\end{array}$ & ------ \\
\hline Popayán & $\begin{array}{l}\text { Muy intenso, se sintió un fuerte } \\
\text { temblor, inmenso número de fieles } \\
\text { que se hallaban en la catedral } \\
\text { corrieron despavoridos, reina inmenso } \\
\text { pánico; la ciudad está alarmadísima. } \\
\text { Ha causado gran consternación, fue } \\
\text { sentido fuertemente. }\end{array}$ & ------ & $\begin{array}{l}\text { La catedral sufrió algunas averías en el } \\
\text { techo. Algunas casas particulares se han } \\
\text { agrietado, la capilla de Belén ha sufrido } \\
\text { varios desperfectos, por el principal } \\
\text { torreón quedó vencida. Causó daños en } \\
\text { la catedral y en otras iglesias, lo mismo } \\
\text { que en la universidad. }\end{array}$ \\
\hline Restrepo & Temblor aquí fue terrible & $\begin{array}{l}\text { Produjo graves pérdidas } \\
\text { y de consideración en } \\
\text { aquellas poblaciones, } \\
\text { particularmente en el } \\
\text { comercio. }\end{array}$ & Varias casas caídas \\
\hline
\end{tabular}

